University of Louisville

ThinkIR: The University of Louisville's Institutional Repository

Electronic Theses and Dissertations

$5-2017$

\title{
A novel MRA-based framework for the detection of changes in cerebrovascular blood pressure.
}

Yitzhak Atakilt Gebru

University of Louisville

Follow this and additional works at: https://ir.library.louisville.edu/etd

Part of the Bioimaging and Biomedical Optics Commons

\section{Recommended Citation}

Gebru, Yitzhak Atakilt, "A novel MRA-based framework for the detection of changes in cerebrovascular blood pressure." (2017). Electronic Theses and Dissertations. Paper 2818.

https://doi.org/10.18297/etd/2818

This Master's Thesis is brought to you for free and open access by ThinkIR: The University of Louisville's Institutional Repository. It has been accepted for inclusion in Electronic Theses and Dissertations by an authorized administrator of ThinkIR: The University of Louisville's Institutional Repository. This title appears here courtesy of the author, who has retained all other copyrights. For more information, please contact thinkir@louisville.edu. 


\title{
A NOVEL MRA-BASED FRAMEWORK FOR THE DETECTION OF CHANGES IN CEREBROVASCULAR BLOOD PRESSURE
}

By

\author{
Yitzhak Atakilt Gebru \\ B.Sc. in Bioengineering, University of Louisville, KY, USA, 2015
}

\begin{abstract}
A Thesis
Submitted to the Faculty of the

J.B. Speed School of Engineering of the University of Louisville

in Partial Fulfillment of the Requirements

for the Degree
\end{abstract}

Master of Engineering in Bioengineering

Department of Bioengineering

University of Louisville

Louisville, Kentucky

May 2017 


\section{A NOVEL MRA-BASED FRAMEWORK FOR THE DETECTION OF CHANGES IN CEREBEROVASCULAR BLOOD PRESSURE}

Submitted by:

Yitzhak Atakilt Gebru

A Thesis Approved on

(Date)

by the Following Reading and Examination Committee:

Ayman El-Baz, Ph.D. Thesis Director

Hermann B. Frieboes, Ph.D.

Andre Faul, Ph.D.

Guruprasad A. Giridharan, Ph.D. 


\section{ACKNOWLEDGMENTS}

There are many people I must thank for the opportunities given to me that have led me to become successful in this endeavor. Throughout my life and academic career, there are many who've supported me. I would like to acknowledge a few who have had the most impact. Above all, I'd like to thank my father, Capt. Atakilt H. Gebru, my mother, Frehiwot Wubshet, and my brother, Simon A. Gebru, for their continual love and support. Specifically, I want to thank my father. None of this would have been possible without his guidance, persistence, and belief in my educational goals.

I would also like to thank my thesis advisor, Dr. Ayman El-Baz. Throughout the past four years, he has mentored me and given me many opportunities to learn and excel. He has constantly supported me in my studies, guided me in challenging times, and always believed in me when I doubted. I have respected him as my professor and now I can also say as my friend. I would also like to thank the rest of my thesis committee, Dr. Frieboes, Dr. Faul, and Dr. Giridharan. Specifically, I would like to add that Dr. Frieboes has been a supportive mentor that has been there to advise me in making smart choices in my academic career. I would also like to thank Dr. Giridharan for going above and beyond in helping me make this thesis be the best it can be.

I would also like to thank Dr. J. Richard Jennings and his research associate Dana Jorgensen, both of the University of Pittsburg's Department of Psychiatry. They answered many questions regarding cardiovascular physiology and provided the imaging and clinical data used in this thesis.

Finally, I would like to thank all the BioImaging Lab members, Dr. Ahmed Soliman, Dr. Marwa Ismail, Mohamed Shehata, Dr. Ahmed Shalaby, Dr. Ali Helmi, Ahmed Shaffie, and anyone else who has ever helped me answer one of my many questions. They have assisted me in writing 
code, solving problems, and pointing me in the right direction when needed. This research could not have been conducted without their support. 


\title{
ABSTRACT
}

\section{A NOVEL MRA-BASED FRAMEWORK FOR THE DETECTION OF CHANGES IN}

\author{
CEREBROVASCULAR BLOOD PRESSURE
}

\author{
Yitzhak Atakilt Gebru
}

April, 2017

Background: High blood pressure (HBP) affects 75 million adults and is the primary or contributing cause of mortality in 410,000 adults each year in the United States. Chronic HBP leads to cerebrovascular changes and is a significant contributor for strokes, dementia, and cognitive impairment. Non-invasive measurement of changes in cerebral vasculature and blood pressure (BP) may enable physicians to optimally treat HBP patients. This manuscript describes a method to non-invasively quantify changes in cerebral vasculature and BP using Magnetic Resonance Angiography (MRA) imaging.

Methods: MRA images and BP measurements were obtained from patients ( $\mathrm{n}=15, \mathrm{M}=8, \mathrm{~F}=7$, Age $=49.2 \pm 7.3$ years) over a span of 700 days. A novel segmentation algorithm was developed to identify brain vasculature from surrounding tissue. The data was processed to calculate the vascular probability distribution function (PDF); a measure of the vascular diameters in the brain. The initial (day 0) PDF and final (day 700) PDF were used to correlate the changes in cerebral vasculature and BP. Correlation was determined by a mixed effects linear model analysis.

Results: The segmentation algorithm had a $99.9 \%$ specificity and $99.7 \%$ sensitivity in identifying and delineating cerebral vasculature. The PDFs had a statistically significant correlation to BP changes below the circle of Willis ( $\mathrm{p}$-value $=0.0007)$, but not significant $(\mathrm{p}$-value $=0.53$ ) above the circle of Willis, due to smaller blood vessels.

Conclusion: Changes in cerebral vasculature and pressure can be non-invasively obtained through MRA image analysis, which may be a useful tool for clinicians to optimize medical management of HBP.

Keywords: LCDG; HBP; segmentation; CDF; MAP; PDF 


\section{TABLE OF CONTENTS}

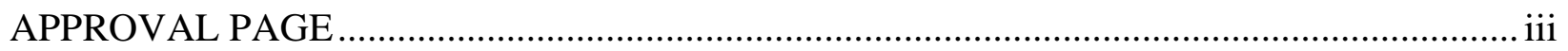

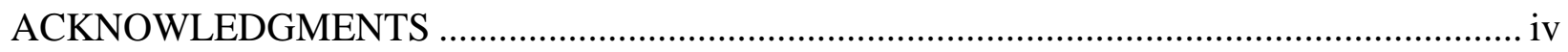

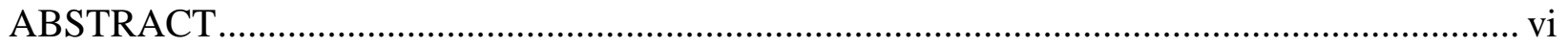

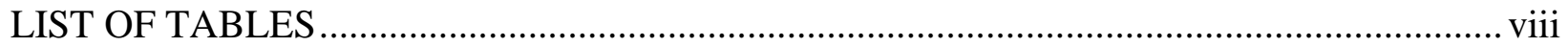

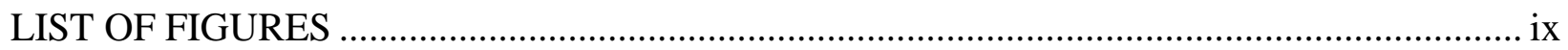

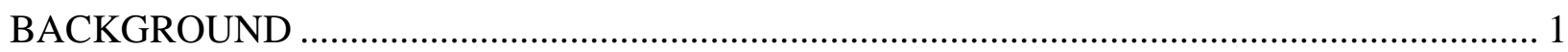

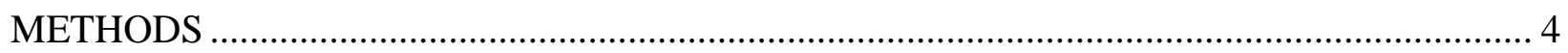

Patient Demographics ............................................................................................. 4

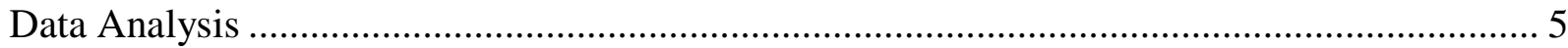

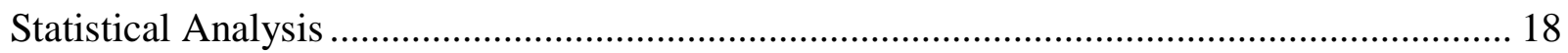

3D Reconstruction of Cerebral Vasculature........................................................................ 18

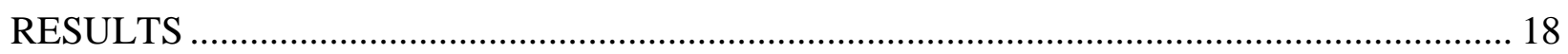

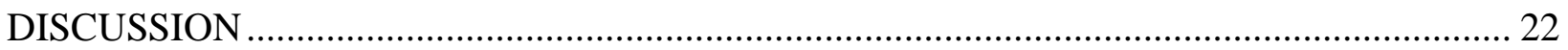

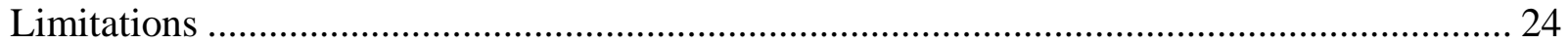

CONCLUSIONS

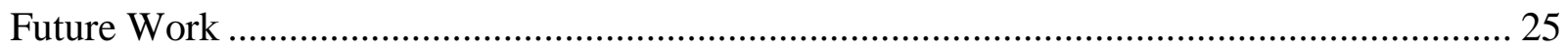

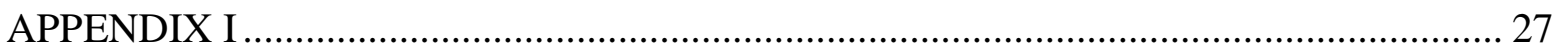

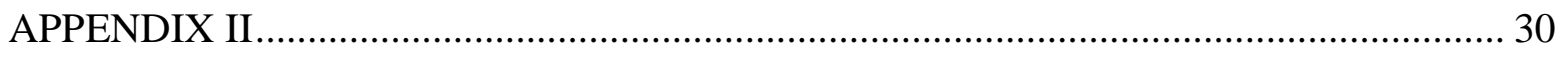

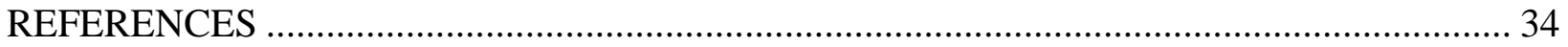




\section{LIST OF TABLES}

Table 1 - Sensitivity and specificity values for automatically segmented images at day 0 , day 700, and both combined.

Table 2 - Mixed effects linear model statistical evaluation. p-values < 0.05 show statistical significance with regards MAP. Diameter denotes size of vasculature in segmentation images. Age, sex, and timepoints are clinically acquired data...................................................... 20

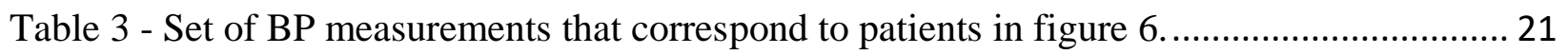




\section{LIST OF FIGURES}

Figure 1 - Hierarchy of stress and its effects.

Figure 2 - Framework of the data analysis procedure for quantifying cerebrovascular changes

from imaging data.

Figure 3 - Original MRA image slice of sample patient at day 0 (left image). Manually

segmented ground truth (GT) image from image on left (right image).

Figure 4 - Illustration shows the segmentation errors between the ground truth, G, and the proposed automatic segmentation, C [5].

Figure 5 - Example of segmentation algorithm output; (a) Image slices of sample patient at day 0 . Automatically segmented from original MRA images by the algorithm based on LCDG determined threshold values from GT slices (b) The 3D reconstruction of the segmented cerebrovascular system.

Figure 6 - Set of graphs depicting various patient CDFs. The graphs indicate the probability that blood vessels may be of a certain diameter or less. The closer to 1 on the y-axis a CDF curve is, the greater the chance for vessels to be that diameter or less. The closer to 0 on the $y$-axis a curve is, the more likely that the diameter of vessels is larger. . 21

Figure 7 - Applying a 3-D growing algorithm to the volume of binary segmented images allows for visualization of the automatically segmented MRA data. Day 0 of patient $C$ (top left). Day 700 of patient C (top right). Day 0 of patient D (bottom left). Day 700 of patient D (bottom right). Some apparent differences between vascular construction in areas below the circle of Willis are highlighted... 


\section{BACKGROUND}

The World Health Organization has proclaimed stress to be the "Health Epidemic of the $21^{\text {st }}$ Century." Stressors have profound impact on mood, behavior, and health. The correlations between stressors and disease can be affected by the nature, number, and persistence of the stressors. The individual's genetics can play a role in how vulnerable they are while psychosocial factors and learned patterns of coping can reduce vulnerability. It has been shown that reducing long term stress can increase quality of life and reduce the course of chronic diseases[1].

Research has shown that excessive amounts of stress and the perception that it impacts health negatively is associated with poor physical and mental health. It was shown that individuals who perceived that stress affects their health negatively and that reported a large amount of stress had an increased risk of premature death[2].

Unlike short-term stress, which can be relieved by directly removing the stressor, chronic long-term stress cannot be relieved by a fight or flight response. This results in harmful reactions by the body and mind to deal with the stress to achieve homeostasis. The mental consequences have been identified to include such things as increases in smoking, substance abuse, accidents, sleep problems, eating disorders, etc. Populations that live in more stressful areas (communities that experience higher rates of natural disasters, divorce, business failures, etc.) smoke more heavily and experience higher mortality from lung cancer and chronic obstructive pulmonary disorder. It has also been proposed that those with chronic stress may use alcohol as selfmedication for disorders such an anxiety or Post-Traumatic Stress Disorder (PTSD)[1].

When the body feels threated, or stressed, the nervous system responds by releasing stress hormones such as adrenaline and cortisol which heighten the body's senses to prepare for emergency action. Senses become sharper, hearth rate increases, blood pressure rises, rate of 
breathing increases, etc. This natural response is important as it can be a lifesaving response in the face of danger. The same response over a long period, however, can result in negative outcomes affecting the cardiovascular system.

The sympathetic nervous system (SNS) stimulated chronically due to stress leads to increases in blood pressure and vascular hypertrophy. The constricting muscles of the vasculature thicken and produce an elevated resting blood pressure. The response stereotypy is also increased which is a tendency to respond to stressors with a vascular response. Chronically elevated blood pressure forces the heart to work harder which will lead to hypertrophy of the left ventricle. In time, the elevated and quickly shifting blood pressure levels will lead to damaged arteries[1].

\section{Stress and Disease}

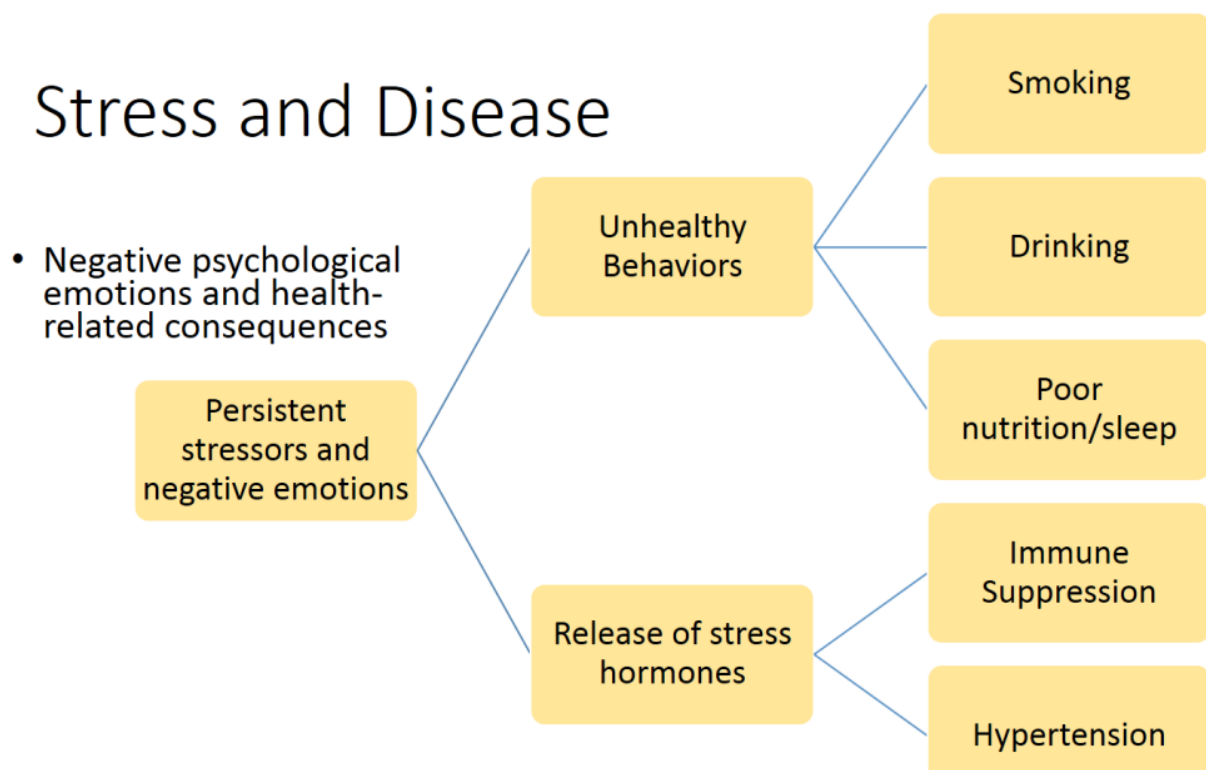

Smoking

Hypertension

Stroke

Figure 1 - Hierarchy of stress and its effects.

Approximately 1 in 3 adults in the U.S. has high blood pressure (HBP). HBP is the primary or contributing cause of mortality in 410,000 adults each year with associated healthcare costs of $\$ 46$ billion[3]. Chronic stress[4], high sodium intake[5], and renal dysfunction[6] are the primary causes for HBP. Chronic HBP, alters the structure of cerebral blood vessels and disrupts cerebral 
vasoregulatory mechanisms. Significantly, HBP induces hypertrophic and eutrophic remodeling in cerebral blood vessels[7]. In hypertrophic remodeling, HBP induces increases in wall thickness and reduction in the vessel lumen in cerebral blood vessels. In eutrophic remodeling, smooth muscles cells undergo a rearrangement that leads to a reduction of the vessel lumen without changes in total vascular mass or wall thickness. These cerebrovascular changes are hypothesized to be a significant contributor for strokes, brain lesions, cerebral ischemic injury, dementia, and cognitive impairment[7-9].

Currently, HBP is diagnosed and medically managed when systemic BP measurements using sphygmomanometer are greater than 140/90 mmHg. However, BP measurement via sphygmomanometer cannot quantify the cerebrovascular or cerebral blood pressure changes that can increase the risk of cerebral adverse events. Importantly, cerebrovascular changes and high cerebral BP are hypothesized to occur prior to the onset of systemic HBP and are thought to be the driver of systemic blood pressure changes[10-12]. Thus, quantification of cerebrovascular and cerebral BP changes may help identify and stratify patients at risk of cerebral adverse events, enable medical treatment prior to the onset of systemic hypertension, and optimize medical management of HBP patients.

Imaging techniques including Magnetic Resonance Imaging (MRI) and Magnetic Resonance Angiography (MRA) scans have been traditionally used in the quantification of organ structural changes[13]. In literature, MRI scanning has been used for volumetric measurement of the ventricular cavities and myocardium[14], and to determine intravascular pressures from MR velocity data in large vessels like the aorta or pulmonary artery [15]. MRA scanning has been used to quantify flow measurements in the collateral arteries of patients with internal carotid artery occlusions[16] but has not been utilized for the estimation of vascular pressure changes in the 
brain, to our knowledge. Detection of cerebrovascular or cerebral BP changes using MRA analysis has not been accomplished due to the lack of accurate segmentation algorithms that can delineate the smaller blood vessels in the brain (in comparison to aorta or pulmonary arteries) from the surrounding soft tissue. Further, there are no methods to quantify cerebrovascular changes and to correlate them to changes in cerebral BP from MRI/MRA imaging. This manuscript presents novel methodologies to delineate cerebral blood vessels from the surrounding tissue, quantify cerebrovascular changes, and correlate the cerebrovascular changes to BP.

\section{METHODS}

The goal of this manuscript is to develop a new MRA-based framework for the detection of changes in Cerebrovascular BP. Patient demographics, and details about the proposed methodology and data analysis are presented next.

\section{Patient Demographics}

This study was approved by the Institutional Review board (IRB) at the University of Pittsburgh. MRA data and BP measurements obtained from patients ( $n=15, M=8, F=7$, Age $=49.2 \pm 7.3$ ) over a 700-day study period were retrospectively analyzed. MRA imaging data and systemic BP measurements were obtained on the same day using a $3 \mathrm{~T}$ Trio TIM scanner using a 12-channel phased-array head coil and using sphygmomanometers, respectively. The volume of scans composed of 3D multi-slab high resolution images with 160 slices, thickness of $0.5 \mathrm{~mm}$, resolution of 384 x 448, a flip angle of 15 degrees, repetition time of $21 \mathrm{~ms}$, and echo time of $3.8 \mathrm{~ms}$.

The subjects had an average day 0 systolic pressure of $122 \pm 6.9 \mathrm{mmHg}$, an average day 0 diastolic pressure of $82 \pm 3.8 \mathrm{mmHg}$, an average day 700 systolic pressure of $118.9 \pm 12.4$ $\mathrm{mmHg}$, and an average day 700 systolic pressure of $79.9 \pm 11.0 \mathrm{mmHg}$. 
The 15 subjects were selected to represent a range of blood pressure changes over the 700 days and the data was analyzed blind to the patient BPs.

\section{Data Analysis}

The analysis of patient MRA data consists of five key steps (Figure 1) consisting of: 1) manual segmentation of training slices to identify ground truth, 2) automatic segmentation for all slices delineates the blood vessels from the surrounding soft tissue by combining the segmented ground truths with the Linear Combination of Discrete Gaussians (LCDG) models for grey level distribution, 3) voxel matching for obtaining temporal subtraction images enhances the ability to see cerebrovascular and the created distance map quantifies the changes in patients between day 0 and day 700,4$)$ generation of a probability distribution function (PDF) which describes the distribution of pixel distances from vascular edges and is used to statistically correlate to $\mathrm{BP}$ and 5) estimation of the cumulative distribution function $(\mathrm{CDF})$ to observe the summated probability of cerebrovascular changes in the same patient from day 0 and day 700 .
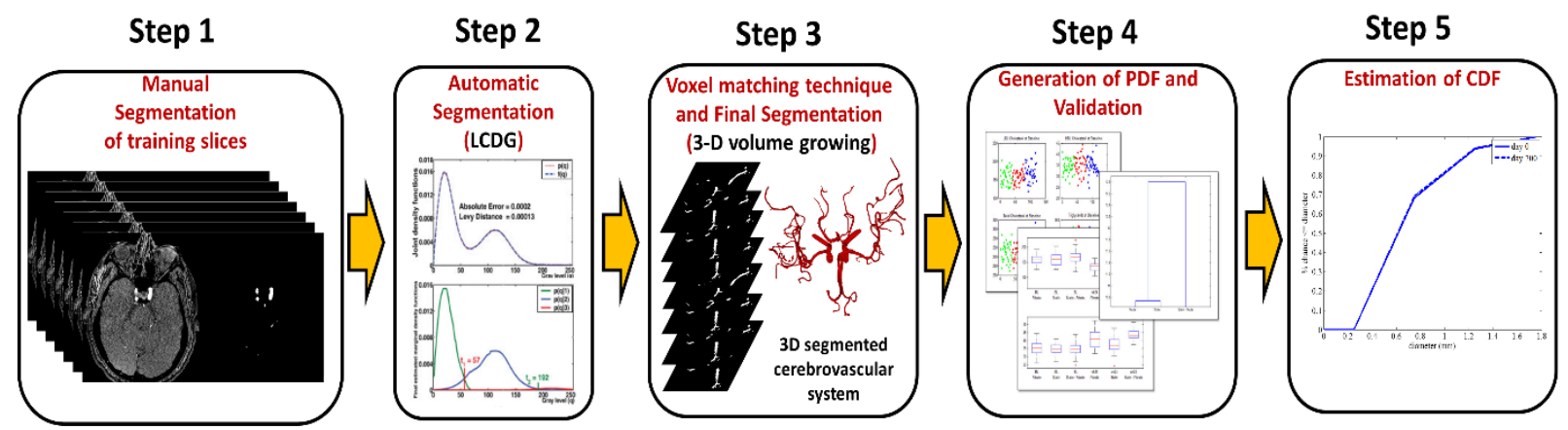

Figure 2 - Framework of the data analysis procedure for quantifying cerebrovascular changes from imaging data.

Manual Segmentation of Training Slices. MRA data from a patient consists of 160 MRA slices. Every tenth slice is manually segmented to extract the blood vessels from surrounding tissue using Adobe Photoshop (Adobe Systems, CA). This methodology 
allows for delineation of the blood vessel from the surrounding tissue at a pixel level accuracy where the largest limitation is the resolution of the MRI machine itself. The manually segmented training binary (black for surround tissue and white for target vasculature) slices are referred to as a ground truths (GT) as the images are correct and free from artifacts or noise (Figure 2). The manual segmentation of select slices is used for the initialization and optimization of the segmentation algorithm. The segmentation algorithm is subsequently used for segmenting all obtained slices.

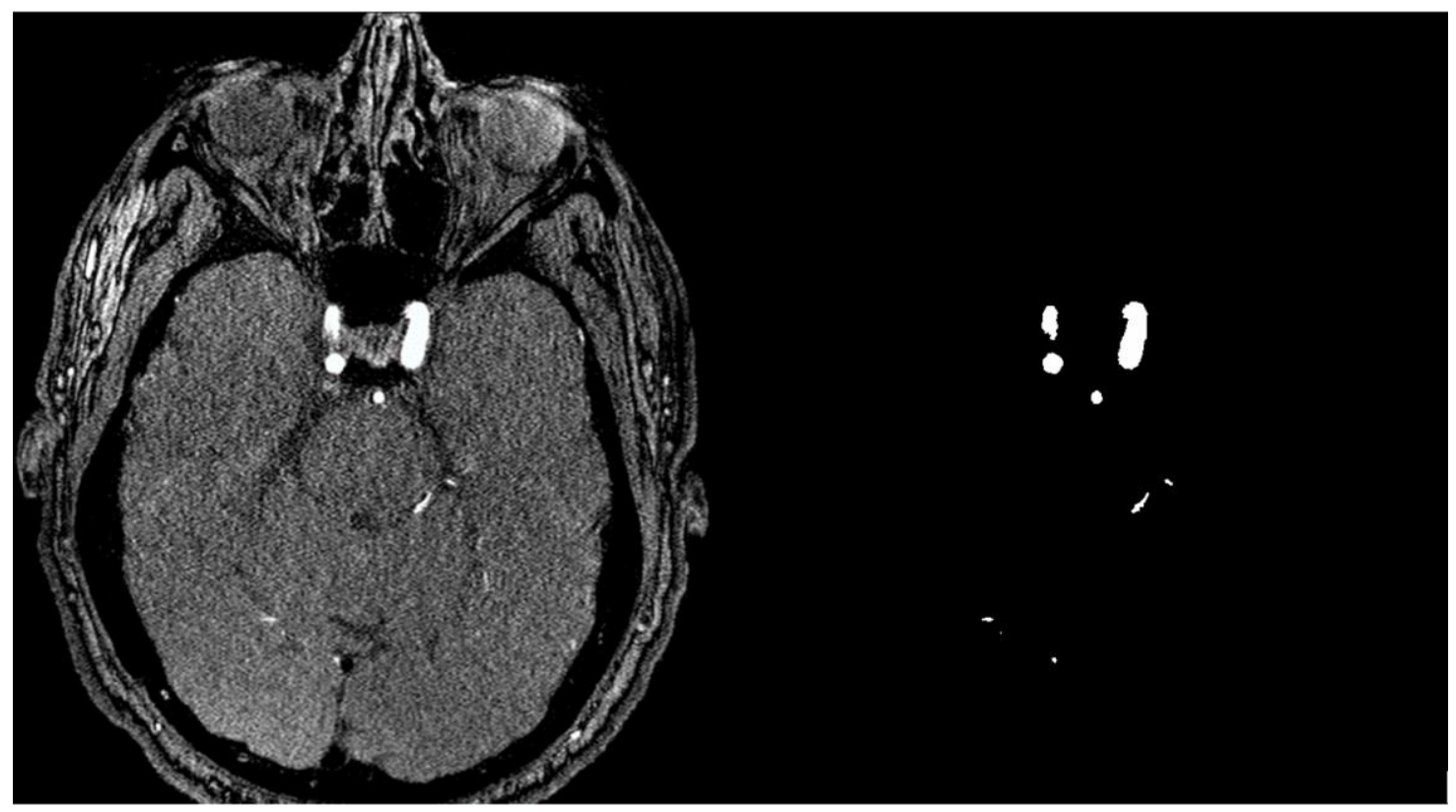

Figure 3 - Original MRA image slice of sample patient at day 0 (left image). Manually segmented ground truth (GT) image from image on left (right image).

Automatic Segmentation. One of the most important problems relating to common computer-assisted diagnostics is the segmentation of accurate $3 \mathrm{D}$ cerebrovascular system information from MRA images. A good choice for the suppression of background signals and for quantifying blood flow velocity vectors for each voxel is phase contrast magnetic resonance angiography (PC-MRA). Time of Flight magnetic resonance angiography (TOFMRA) is fast and provides images with high contrast but is less quantitative. Statistical, 
deformable, scale-space filtering, centerline-based, and hybrid are some of the more popular methods for extracting vasculature structures from MRA data.

Entwining an image with Gaussian filters at multiple scales, a process referred to as multiscale filtering, enhances curvilinear structures in 3-D medical images. The eigenvalues of the Hessian at each voxel are inspected to conclude the 3D structures' local shapes (because of the eigenvalues, blood vessels which are linear structures will differ from plane structures, unstructured objects, and speckle noise). The output of the multiscale filter output forms a new image in which the curvilinear structures become brighter whereas other parts (planar structure and speckle noise for example) become darker[17]. Segmentation and visualization can be directly implemented on such an image using a deformable model. The eigenvalues can also define a set of voxels relating to the centerlines of the target vasculature[18]. The multiscale filter responses at each of the voxel sets will decide the probability that a voxel belongs to a vessel of a specific diameter. Each voxel has an assigned max response over all diameters. From the estimated centerlines and diameters, a surface model of the vasculature is reconstructed. Post segmentation of the filtered MRA image using thresholding, anisotropic diffusion techniques preserve small vessels and remove noise[19]. A multiscale technique based on Markov marked point processes to extract coronary vasculature from X-Ray angiograms was proposed by [20]. The coronary vessels would be modeled locally as piece-wise linear segments of varying widths, lengths, orientations, and locations. The centerlines of the vasculature are isolated using a Markov object process specified by a uniform Poisson process.

Representing two-point centerline extractions as the minimum cost integrated along the centerline path can be formulated with centerline minimal path-based techniques. A 
study used multi-scale medialness filters to compute the cost of graph edges in a graphbased minimal path detection method to extract the vessels' centerlines. Considering the length and scale of the centerlines of the vessels, a post processing step was conducted to extract a full tree of vasculature centerlines[21]. Another study used the shortest paths to extract tubular structures from 2-D images by means of an automatic framework. The framework used orientation and multiscale optimization to propagate 4-D (orientation, space, scale) paths onto 2-D images[22]. Another study took the 3-D model and reduced it to a curve, albeit in 4-D, with an extra dimension of the non-spatial variety that describe the thickness of the vessel. A minimal path approach was used to find the shortest path between user defined end points on the 4D curve. Simultaneously, the detected path described both the surface and the vessel centerline[23]. Extending that approach, another study looked at considering vessel orientation based on an anisotropic optimally oriented flux (OOF) descriptor[24]. A problem for shortest path techniques is that a false straight shortcut path might occur instead of following the vessel. To overcome that issue, another study employed the use of a minimum average-cost path model to look at CT images and segment the 3D coronary arteries from them. The average edge cost was reduced along paths in the 4-D graph constructed by voxels and related radii[25].

The approach in that which one approximates the boundary surface of the vasculature is referred to as a deformable model. An example of one method involves a 'snake'; the initial boundary which evolves for the sake of optimization of the surface energy. That surface energy will depend on the smoothness of the surface and on image gradients[26, 27]. A study by Xu and Prince used a gradient vector flow (GVF) field to steer snakes into the concavities of objects. This increased the capture range of the evolving 
boundary[27]. Guided by the GVF field, the deformable model has been employed in the segmentation of 2D and 3D computer tomographic angiography (CTA)[28] and 3D MRA[25]. Deformable models are made more efficient for segmentation of vessels in the brain when using topologically adaptable surfaces[29]. Geodesic active contours[30] implemented with level set techniques offer flexible topological adaptability to segment the MRA images[26] including more efficient adaptation to local geometric structures represented (tensor eigenvalues, for example[31]). Quick segmentation of blood vessel surfaces is obtained by inflating a 3D balloon with fast marching methods[32]. The twostep segmentation of a 3-D vascular tree in[33] is first carried out locally in a small volume of interest. A global topology is then estimated to initialize a new volume of interest.

Holtzman-Gazit et al.[34] extracted vasculature in CTA images based on varying principles. The framework they used combined the Chan-Vese minimal variance model with a geometric edge alignment measure and the geodesic active surface model. Manniesing et al.[33] proposed a level set-based vascular segmentation method for finding vessel boundaries in CTA images. The level set function is attracted to the vessel boundaries based on a dual object (vessels) the distribution of background intensity, which are estimated from the intensity histogram.

Forkert et al.[35] employed the use of a vesselness filter to direct a level set to extract vessels from TOF-MRA data. Deformable models produce much better results from experiments compared to scale-space filtering, however, they both share a common drawback; manual initialization. They are also both slow when compared to statistical approaches. 
Extracting the statistical data from a vascular tree is a completely automatic process. The accuracy, however, depends on the framework of the probability models. The MRA images are multi-modal so that the intensity or gray-level signals in the target region (blood vessels, brain tissue, etc.) is related to a dominant mode of the sum marginal probability of the signals[36]. Extraction of blood vessels via adaptive statistical approaches were proposed by [37] for the PC-MRA data and [38] for the TOF-MRA data. In [38] the Gaussians were replaced with a more suitable Rician distribution. The marginal data distribution was represented in [37] with two Gaussians and one component that is uniform for the motionless cerebrospinal fluid (CSF), arteries, and brain tissue. To estimate all the relevant parameters, an expectation-maximization (EM) algorithm was used in both studies. For the TOF-MRA data set, a "modified EM" was used by replacing gray levels in pixels considered by their starting EM arrangement with a small gray level distribution. According to Webb [39], however, the modified EM includes changes that make it more similar to the classic ones used for many years for the estimation of density. It makes it so that pixels appeared in their starting arrangement as an exact copy of a general EM framework.

There have been attempts to combine parts of these mentioned approaches to create hybrids. A region-based deformable contour for the segmentation of cylindrical objects was formulated in [40] through a combination of shape information and signal statistics. Another hybrid approach, used by [41], lead a deformable surface model second order intensity statistics and surface geometry to segment vasculature from PC- and TOF-MRA images. To repeatedly extract vasculature from images acquired via rotational angiography, a hybrid of a Gaussian statistical model with the maximum intensity 
projection (MIP) images need to be collected at three orthogonal directions [42]. As an alternative to that, [43] used an extraction of the object boundaries by fusing an iterative thresholding approach with component label analysis and region growing.

These previously used approached have several limitations, however.

1 - Most can only be applied to 1 image type; TOF- or PC-MRA.

2 - To initialize a vessel of interest, a designation is required by the user.

3 - Some of the models require an assumption that the patient has uniformly circular vessels. These models would not hold true for patients with irregular vessel shapes, such as those with an aneurysm of a stenosis.

4 - Besides the statistics approaches, the other models require much processing power and time to complete analysis.

5 - The statistical approaches use predefined probability models. These models wouldn't fit all cases because intensity distributions of blood vessels can vary between patients, scanning apparatus, and the parameters used[36].

Our approach is to rapidly and accurately extract the blood vessel data by defining the probability models of each region of interest within the statistical approach and not predefining the probability models[36-38]. The empirical gray level distribution for each MRA slice is closely approximated with a LCDG, then it is split into three individual LCDGs, one per region of interest. These regions are associated with three dominant modes: darker bones and fat, gray brain tissues, and bright blood vessels, respectively. The models identified specify an intensity threshold for extracting blood vessels in that slice. A 3-D connectivity filter is then applied to the extracted voxels (voxel = volume $\mathrm{x}$ element; a representation for a 3-D pixel) to select the desired vascular tree. This method results in 
more precise region models with better segmentation accuracy compared to other methods[36].

Adapting the EM based technique to the LCDG allows for precise identification of the LCDG-model including the number of its positive and negative components[44] and for identification of a continuous LCDG-model that contains the probability distribution.

An expected log-likelihood is used as a model identification criterion in [36]. Let $\mathrm{X}=(\mathrm{Xs}: s=1, \ldots, S)$ denote a 3-D MRA image containing $\mathrm{S}$ co-registered 2-D slices $\mathrm{X}_{\mathrm{s}}=$ $\left(X_{S}(i, j):(i, j) \in R ; X_{s}(i, j) \in Q\right)$. Here, $R$ and $Q=\{0,1, \ldots, Q-1\}$ are a rectangular arithmetic lattice supporting the 3-D image and a finite set of Q-ary intensities (gray levels), respectively. Let $F_{s}=\left(f_{s}(q): q \in Q ; \sum_{q} \in Q\right.$ fs $(q)=1$, where $q$ denotes the gray level, be an empirical marginal probability distribution of gray levels for the MRA slice $X_{\mathrm{s}}$.

In accordance with [44], each such slice is considered as a $K$-modal image with a known number $K$ of the dominant modes related to the regions of interest. To segment the slice by separating the modes, an estimation of the individual probability distributions of the signals associated with each mode from $\mathrm{F}_{\mathrm{s}}$ is necessary. In contrast to a conventional mixture of Gaussians, one per region, or slightly more flexible mixtures involving other simple distributions, one per region, we closely approximate $F_{s}$ with LCDG. The LCDG of the image is then partitioned into submodels related to each dominant mode $[27,34,35]$.

The discrete Gaussian (DG) is defined as the probability distribution $\Psi_{\theta}=(\psi(\mathrm{q} \mid \theta)$ : $\mathrm{q} \in \mathbf{Q})$ on $\mathbf{Q}$ of gray levels such that each probability $\psi(\mathrm{q} \mid \theta)$ relates to the cumulative Gaussian probability function $\Phi_{\theta}(\mathrm{q})$ as follows (here, $\theta$ is a shorthand notation for the mean, $\mu$, and variance, $\sigma^{2}$, where $\left.\theta=\left(\mu, \sigma^{2}\right)\right)$ : 


$$
\psi(\mathrm{q} \mid \theta)=\left\{\begin{array}{cl}
\Phi_{\theta}(0.5) \text { for } \mathrm{q}=0 & \text { for } \mathrm{q}=1 \\
\Phi_{\theta}(\mathrm{q}+0.5)-\Phi_{\theta}(\mathrm{q}-0.5) & \text { for } \mathrm{q}=1, \ldots, \mathrm{Q}-2 \\
1-\Phi_{\theta}(\mathrm{Q}-1.5) & \text { for } \mathrm{q}=\mathrm{Q}-1
\end{array}\right.
$$

The LCDG with $C_{p}$ positive and $C_{n}$ negative components such that $C_{p} \geq K$

$$
p_{\boldsymbol{w}, \boldsymbol{\Theta}}(q)=\sum_{r=1}^{C_{p}} w_{p, r} \psi\left(\mathrm{q} \mid \theta_{\mathrm{p}, \mathrm{r}}\right)-\sum_{l=1}^{C_{n}} w_{n, l} \psi\left(\mathrm{q} \mid \theta_{\mathrm{n}, \mathrm{l}}\right)
$$

has obvious restrictions on its weights $\mathbf{w}=\left[\mathrm{w}_{\mathrm{p}}, ., \mathrm{w}_{\mathrm{n}},.\right]$, namely, all the weights are nonnegative and

$$
\sum_{r=1}^{C_{p}} w_{p, r}-\sum_{l=1}^{C_{p}} w_{n, l}=1
$$

Generally, the true probabilities are nonnegative: $\mathrm{p}_{\mathbf{w}, \boldsymbol{\Theta}}(\mathrm{q}) \geq 0$ for all $\mathrm{q} \in \mathbf{Q}$. Therefore, the probability distributions comprise only a proper subset of all the LCDGs in (1), which may have negative components $\mathrm{p}_{\mathbf{w}, \boldsymbol{\Theta}}(\mathrm{q})<0$ for some $\mathrm{q} \in \mathbf{Q}$.

Our goal is to find a K-modal probability model that closely approximates the unknown marginal gray level distribution. Given $\mathbf{F}_{\mathrm{s}}$, its Bayesian estimate $\mathbf{F}$ is as follows[39]: $\mathrm{f}(\mathrm{q})=\left(|\mathbf{R}| \mathrm{f}_{\mathrm{s}}(\mathrm{q})+1\right) /(|\mathbf{R}|+\mathrm{Q})$, and the desired model has to maximize the expected log-likelihood of the statistically independent empirical data by the model parameters:

$$
L(\boldsymbol{w}, \boldsymbol{\Theta})=\sum_{\mathrm{q} \in \mathbf{Q}} \mathrm{f}(\mathrm{q}) \log p_{\boldsymbol{w}, \boldsymbol{\Theta}}(q)
$$

The entire segmentation algorithm is as follows[36].

1) For each successive MRA slice $\mathbf{X}_{\mathrm{s}}, \mathrm{s}=1, \ldots . \mathrm{S}$, 
a) First is to gather the marginal empirical probability distribution $F_{s}$ of gray levels.

b) Find a starting LCDG-model that is nearing Fs by using the initializing algorithm to approximate the values of $\mathrm{C}_{\mathrm{p}}-\mathrm{K}, \mathrm{C}_{\mathrm{n}}$, and the parameters $\mathbf{w}, \boldsymbol{\Theta}$ (weights, means, and variances) of the negative and positive discrete Gaussians (DG).

c) Refine the LCDG-model with the fixed $C_{p}$ and $C_{n}$ by manipulating the other parameters with the modified EM algorithm.

d) Separate the final LCDG-model into K submodels, one for each dominant mode, by minimizing the predicted errors of misclassification and select the LCDG-submodel with the greatest average value (i.e., the submodel linking to the brightest pixels) as the model of the desired vasculature.

e) Extract the blood vessels' voxels in the MRA slice using intensity threshold $t$ separating their LCDG-submodel from the background ones.

2) Eliminate artifacts from the whole set of the extracted voxels with a connection filter that chooses the greatest connected tree system built by a 3-D growing algorithm[45]. 
Algorithm 1 summarizes the adopted segmentation approach:

Algorithm 1 Main Steps of the Segmentation Approach

For each slice $\mathbf{X}_{\mathbf{s}}$, do the following:

\section{LCDG Initialization:}

- Find the marginal empirical probability distribution of gray levels $\mathbf{F}_{\mathbf{s}}$.

- Estimate $C_{p}-K, C_{n}, \mathbf{W}$, and $\Theta$ of the positive and negative DGs.

- Find the initial LCDG-model that approximates $\mathbf{F}_{\mathbf{s}}$.

\section{2- LCDG Refinement:}

- Refine the LCDG-model with the fixed $C_{p}$ and $C_{n}$ by manipulating the other parameters with the modified EM algorithm.

\section{3- Initial Segmentation:}

- Split the final LCDG-model into K submodels by minimizing the expected errors of misclassification.

- Select the LCDG-submodel with the largest mean value as the model of the desired vasculature.

- Extract the blood vessels' voxels in the MRA slice using intensity threshold $t$ separating their LCDG-submodel from the background ones.

\section{4- Final Segmentation:}

- Eliminate artifacts from the whole set of the extracted voxels with a connection filter that chooses the greatest connected tree system built by a 3-D growing algorithm. 
The aim for this procedure is to decipher the threshold for each MRA slice that will allow the full extraction of the bright blood vessels while removing the darker unwanted tissue while also separating surrounding non-vasculature tissue that may be of similar brightness and along the same boundaries. Step 1b's initialization creates the LCDG with the non-negative starting probabilities $\mathrm{p}_{\mathrm{w}, \Theta}(\mathrm{q})$. The refinement in $1 \mathrm{c}$ increases the likelihood, but the probabilities continue to be non-negative. The experiments presented in [36] show the opposite situations were never met.

The automatic segmentation's accuracy is evaluated by calculating total error compared to the ground truths. True positive (TP), true negative (TN), false positive (FP), $\&$ false negative (FN) segmentations are measured for evaluation.

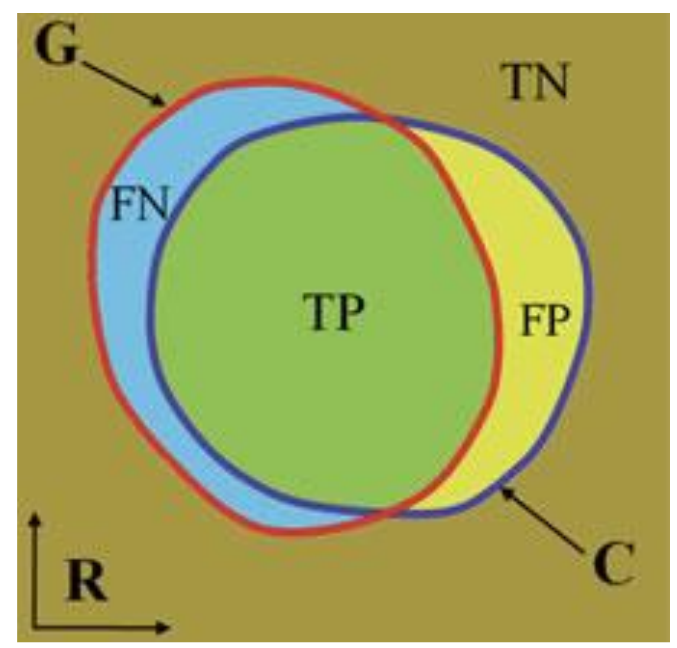

Figure 4 - Illustration shows the segmentation errors between the ground truth, G, and the proposed automatic segmentation, C [5].

From Figure 4, $\mathbf{C}, \mathbf{G}$, and $\mathbf{R}$ denotes the segmented region, the referenced "ground truth", and the whole image frame, respectively. Then, the TP $=|\mathbf{C} \cap \mathbf{G}|$, the TN $=\mid \mathbf{R}-\mathbf{C}$ $\mathbf{U G} \mid$, the $\mathrm{FP}=|\mathbf{C}-\mathbf{C} \cap \mathbf{G}|$; and the $\mathrm{FN}=|\mathbf{G}-\mathbf{C} \cap \mathbf{G}|$. The total error is defined as $\varepsilon=$ $(\mathrm{FN}+\mathrm{FP}) /(\mathrm{TP}+\mathrm{FN})=(\mathrm{FN}+\mathrm{FP}) / \mathrm{G}[46]$. 
Voxel Matching. Voxels are an array of volume elements that constitute a notional three-dimensional space. A 3D affine registration is used to handle the pose, orientation, and the data spacing changes and other scanning parameter changes between day 0 and day 700[47]. In this step, the determined Euclidian radii are converted into diameter values. The output is then converted into a distance map.

Generation of Probability Distribution Function and Validation. The EM-based technique is adapted to the LCDG-model and the distribution of pixel distances is extracted from the distance map to calculate the probability distribution of the cerebrovascular changes. The PDF marks the distribution of white pixels as a true value and black pixels being ignored for the data set. The diameters of the blood vessels are determined by estimating Euclidian center point distances from the edge of a vessel. The data points in the generated PDFs are then extracted and compared to the blood pressure data using statistical analysis.

Calculation of Cumulative Distribution Function. The integral of the PDF is used to generate the $\mathrm{CDF}$ (The $\mathrm{CDF} \mathrm{F}_{\mathrm{X}}$ of a random variable $\mathrm{X}$ is calculated from its $\mathrm{PDF} \mathrm{f}_{X}$ using $\left.F_{X}(x)=\int_{-\infty}^{x} f_{X}(t) d t\right)$ The CDF shows the total summated probability that a blood vessel will take a value less than or equal to a diameter value. It shows the cumulative distribution of the PDF with an upper limit of 1 . The more quickly the CDF line approaches 1, the more certain that the diameter of the blood vessel is smaller compared to a CDF that takes longer to approach 1 . This is illustrated in the results section. 


\section{Statistical Analysis}

Statistical analysis was performed using R software, version 3.3. A mixed effects linear model was used to test the relationship of MRA data with clinical BP measurements. Brain slices were separated into upper (above circle of Willis) and lower (below circle of Willis) compartments to determine correlation with clinical BP readings. The circle of Willis, near the brain base, is where the intracranial cerebral arteries take off from and give rise to progressively smaller vessels[7]. The BP measurements were combined into a single value, the estimated mean arterial pressure $\mathrm{MAP}=(2 \times \mathrm{DBP}+\mathrm{SBP}) / 3$, which was a covariate in the model. Also included in the model were patient age, gender, and a random intercept per patient. The dependent variable was the mean of the Euclidean distance map over the entire vascular tree within each compartment. (Two separate models were fit to the upper and lower compartments.) Statistical significance of fixed effects in the fitted models was determined using likelihood ratio chi-square tests.

\section{$\underline{\text { 3D Reconstruction of Cerebral Vasculature }}$}

A growing tree model, that eliminates any unwanted segmented voxels by choosing the greatest connected vascular tree system, coupled with a smoothing algorithm, was used to generate a 3-D model based on segmented slices [45]. An example for the resultant vascular system is visualized and illustrated in the results section.

\section{RESULTS}

Specificity and sensitivity values were obtained from the segmented images as shown below in Table 1 . The automatically segmented slices for all 15 patients were compared to the manually segmented GTs to determine accuracy of algorithm (Figure 5). The segmentation 
algorithm resulted in the cumulative sensitivity of $0.997 \pm 0.008$ (sensitivity range $=0.969$ to 1 ) and the cumulative specificity of $0.9998 \pm 0.0001$ (specificity range $=0.9994$ to 1 ).

The results of the statistical analysis revealed inverse statistical significance with vessels below the circle of Willis ( $\mathrm{p}$-value $=0.0007)$ with respect to MAP (table 2). The PDF below circle of Willis was not statistically significant based on the age or gender of the patient. The PDF of vessels above circle of Willis only showed statistical significance with age ( $\mathrm{p}$-value $=0.0005)$ (table 2).

In the analysis, 13 out of 15 patients showed correlation between BPs and vessel diameters, however, 2 patients did not show correlation. A depiction of multiple CDFs from below the circle of Willis from 4 patients (A, B, C, and D) are depicted along with the corresponding BPs (Figure 6, Table 3). Patients A and B (Figure 6, Table 3) are examples from the 13 patients showing correlation and patients $\mathrm{C}$ and $\mathrm{D}$ (Figure 6, Table 3) are outliers that did not show correlation.

Patient $\mathrm{C}$ shows a shift in $\mathrm{CDF}$ in the opposite direction of $\mathrm{BP}$ changes, and patient $\mathrm{D}$ shows a large shift in CDF compared to the BP change (Figure 6 - C, Figure 6 - D). The 3-D cerebrovascular model reconstruction of patient C (Figure 6 - C, Figure 7) and patient D (Figure 6 - D 7) indicated significant vascular changes between day 0 and day 700.

Table 1 - Sensitivity and specificity values for automatically segmented images at day 0, day 700, and both combined.

\begin{tabular}{|c|c|c|}
\hline Time & Sensitivity & Specificity \\
\hline Day 0 & $0.997 \pm 0.006$ & $0.9998 \pm 0.0001$ \\
\hline Day 700 & $0.996 \pm 0.008$ & $0.9998 \pm 0.0001$ \\
\hline & & \\
Cumulative & $0.997 \pm 0.008$ & $0.9998 \pm 0.0001$ \\
\hline
\end{tabular}



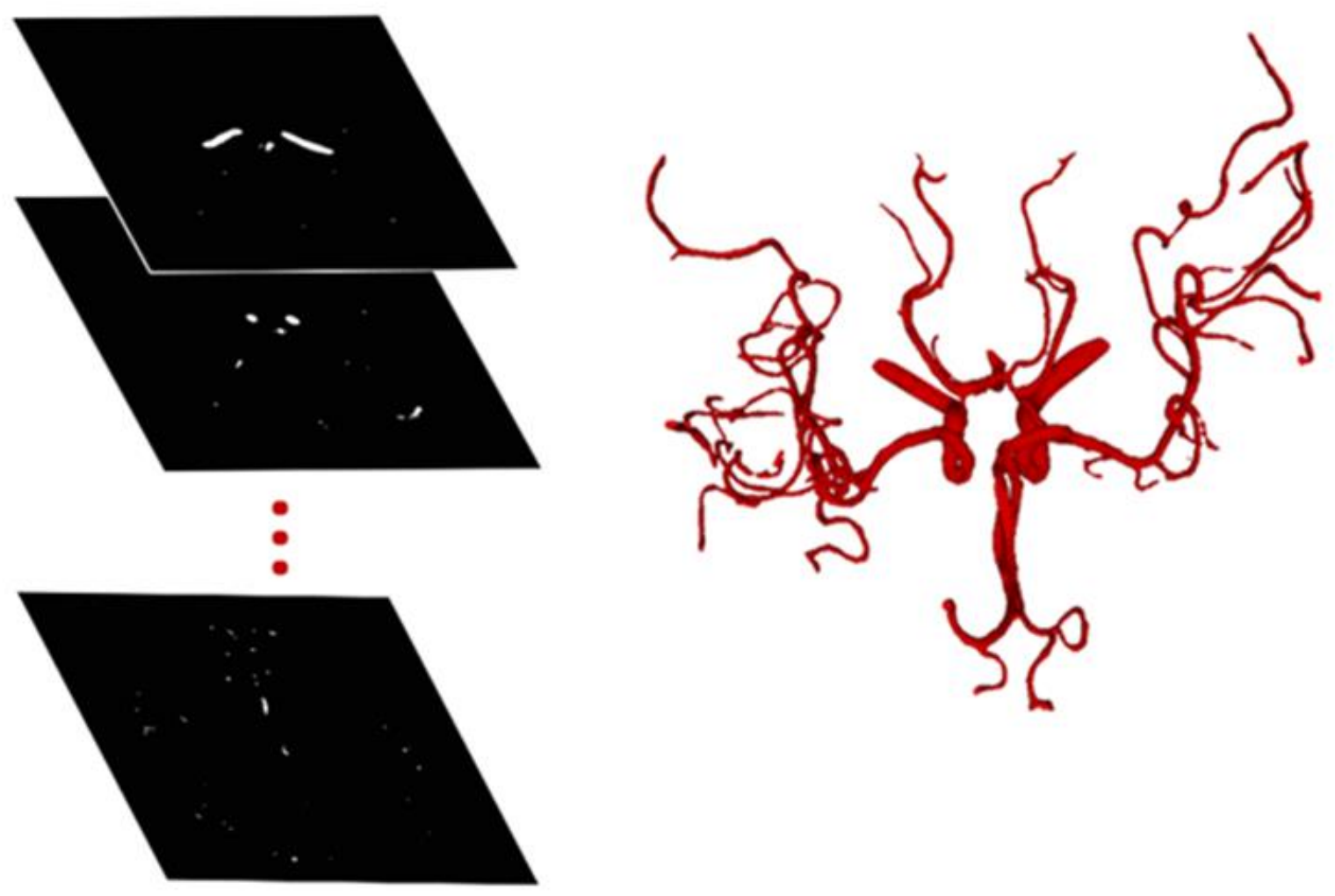

(a)

(b)

Figure 5 - Example of segmentation algorithm output; (a) Image slices of sample patient at day 0. Automatically segmented from original MRA images by the algorithm based on LCDG determined threshold values from GT slices (b) The $3 D$ reconstruction of the segmented cerebrovascular system.

Table 2 - Mixed effects linear model statistical evaluation. p-values $<0.05$ show statistical significance with regards MAP. Diameter denotes size of vasculature in segmentation images. Age and sex are clinically acquired data.

\begin{tabular}{|c|c|c|c|}
\hline \multirow{2}{*}{} & \multicolumn{3}{|c|}{ Mean Diameter of Vessels below circle of Willis } \\
\cline { 2 - 4 } & Effect & $\chi^{2}$ & $p$-value \\
\hline Age & $3.2 \mu \mathrm{m} / \mathrm{y}$ & 0.356 & 0.551 \\
\hline Gender & $\mathrm{F}>\mathrm{M} \mathrm{by} 12.8 \mu \mathrm{m}$ & 0.026 & 0.872 \\
\hline Mean Arterial Pressure & $-5.3 \mu \mathrm{m} / \mathrm{mmHg}$ & 11.63 & 0.0007 \\
\hline & \multicolumn{3}{|c|}{ Mean Diameter of Vessels above circle of Willis } \\
\cline { 2 - 4 } & Effect & $\chi^{2}$ & $p$-value \\
\hline Age & $-16.5 \mu \mathrm{m} / \mathrm{y}$ & 12.29 & 0.0005 \\
\hline Gender & $\mathrm{F}>\mathrm{M} \mathrm{by} 16.0 \mu \mathrm{m}$ & 0.199 & 0.655 \\
\hline Mean Arterial Pressure & $1.6 \mu \mathrm{m} / \mathrm{mmHg}$ & 0.402 & 0.525 \\
\hline
\end{tabular}



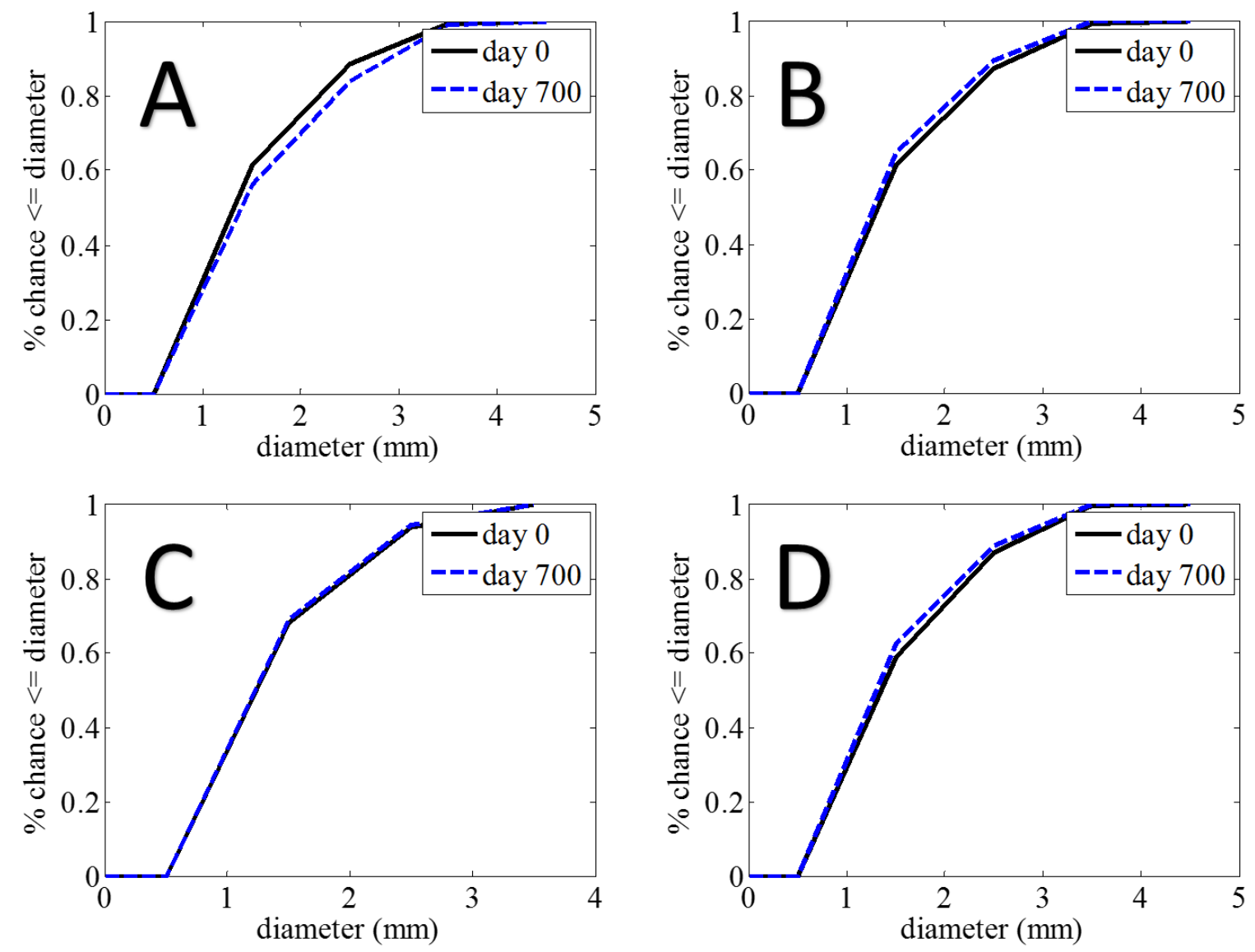

Figure 6 - Set of graphs depicting various patient CDFs. The graphs indicate the probability that blood vessels may be of a certain diameter or less. The closer to 1 on the y-axis a CDF curve is, the greater the chance for vessels to be that diameter or less. The closer to 0 on the $y$ axis a curve is, the more likely that the diameter of vessels is larger.

Table 3 - Set of BP measurements that correspond to patients in figure 6.

\begin{tabular}{|c|c|c|c|c|c|c|}
\hline Patient & \multicolumn{3}{|c|}{ Day 0 } & \multicolumn{3}{c|}{ Day 700 } \\
\hline & $\begin{array}{c}\text { systolic } \\
\text { BP }\end{array}$ & $\begin{array}{c}\text { diastolic } \\
\text { BP }\end{array}$ & MAP & $\begin{array}{c}\text { systolic } \\
\text { BP }\end{array}$ & $\begin{array}{c}\text { diastolic } \\
\text { BP }\end{array}$ & MAP \\
\hline A & 120 & 80.5 & 93.7 & 103.5 & 66.5 & 78.8 \\
\hline B & 130.5 & 83 & 98.8 & 143.5 & 94 & 110.5 \\
\hline C & 118 & 80.5 & 93 & 105.3 & 69 & 81.1 \\
\hline D & 114 & 84.5 & 94.3 & 120 & 88 & 98.7 \\
\hline
\end{tabular}



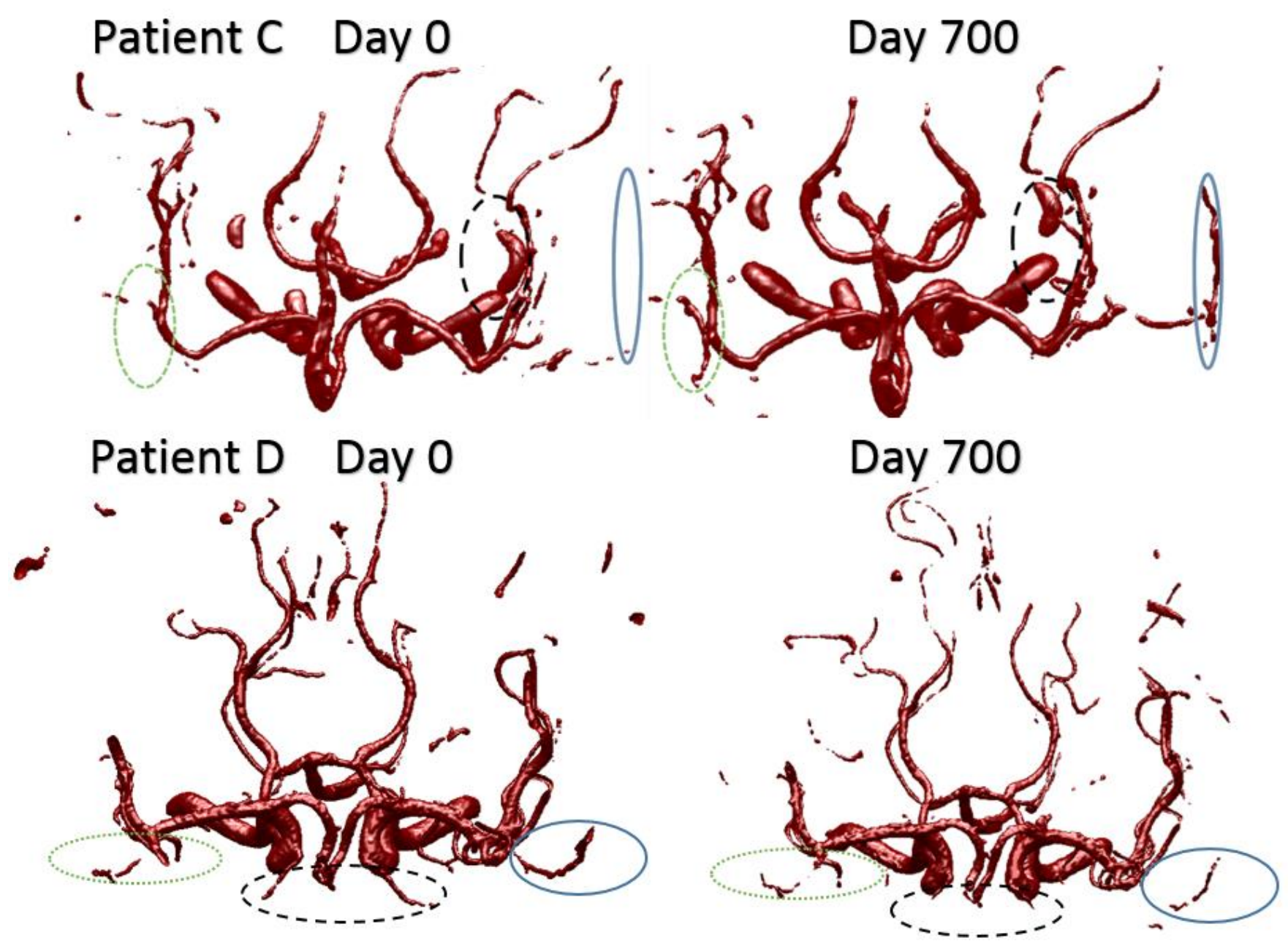

Figure 7 - Applying a 3-D growing algorithm to the volume of binary segmented images allows for visualization of the automatically segmented MRA data. Day 0 of patient $C$ (top left). Day

700 of patient $C$ (top right). Day 0 of patient D (bottom left). Day 700 of patient D (bottom right). Some apparent differences between vascular constructions in areas below the circle of Willis are highlighted.

\section{DISCUSSION}

The average cumulative segmentation algorithm's sensitivity was $0.997 \pm 0.008$ and the specificity was $0.9998 \pm 0.001$. This high level of accuracy shows the benefit of using a manual input to initialize automatic segmentation. Manual segmentation alone would be too time intensive to be used in a practical healthcare setting while using automatic segmentation alone would not 
provide sufficient segmentation accuracy to delineate and quantify the diameters of the smaller cerebral blood vessels. Our segmentation algorithm combines the accuracy of manual segmentation with the benefit of automated and less time intensive approach and provides segmentation with a high degree of accuracy while also minimizing the required time and effort.

The high degree of sensitivity and specificity of our approach in accurately delineating blood vessels from surrounding brain tissue enables the quantification of cerebrovascular changes. The PDFs show the total blood vessel diameter change in time from day 0 and day 700 . Below the circle of Willis, there is a statistical correlation between PDFs and BPs ( $\mathrm{p}$-value $=0.0007$ ), demonstrating that the MAP decreases with increase in average vessel diameter and PDF. The non-invasive PDF data correlates well with most patients' BP measurements below the circle of Willis. Given that cerebral hypertension is hypothesized to precede systemic hypertension[10], our methodology presents a unique tool for potentially initiating early treatment of systemic HBP. The correlation of PDF to MAP was independent of patient gender. The difference in PDF of vessels above circle of Willis was statistically significant with age, which indicates that older patients have constricted cerebral vessels, which may put them at a higher risk of strokes.

In some patients (patients $\mathrm{C}$ and $\mathrm{D}$ ) the change in $\mathrm{CDF}$ did not correlate to changes in MAP or $\mathrm{BP}$, which may indicate impaired auto regulation of cerebral blood flow potentially due to cerebrovascular remodeling [7, 10]. The 3-D cerebrovascular model reconstruction of these patients demonstrated significant vascular changes between day 0 and day 700 . These results demonstrate that drug therapies prescribed using systemic BP alone may not provide optimal medical management. Lack of correlation between CDF and MAP may indicate a higher risk of cerebrovascular adverse events which necessitates more frequent monitoring and/or optimization 
of medical management despite having normal systemic BPs. Using a combination of BP and CDF changes may help minimize the occurrence of adverse events.

\section{$\underline{\text { Limitations }}$}

While our segmentation algorithm significantly improves on automatic segmentation methodologies, it is limited by the resolution limit of the MRI machine performing the MRA scanning. The CDF diameters (Figure 5) start at $0.5 \mathrm{~mm}$ because the distance map calculations determine radius from the edge of a blood vessel and a pixel in the MRA imaging represented $0.25 \mathrm{~mm}$. Any value less than that would not be accurately represented due to the resolution limit. Subsequently, the accuracy of the statistical analysis decreases with decreasing blood vessel size (smaller blood vessels above the circle of Willis).

There were also various over-the-counter medications, vitamins, and supplements the subjects were on during the duration of the study. Tylenol, Aleve, Ibuprofen, Motrin, Omeprazole, and Bayer were some of the over-the-counter medications the subjects used. Supplements such as Calcium, Magnesium, and Vitamin C were also being used during the study. It's unlikely that blood pressures would be affected by these supplements and medications, but there are occurrences of that happening with drugs such as ibuprofen. Further research involving the effects of various medications on cerebrovascular structure is needed. 


\section{CONCLUSION}

Changes in cerebral vasculature and blood pressure can be non-invasively obtained through MRA image analysis. The improved segmentation algorithm coupled with the calculation of CDFs and PDFs can indicate cerebrovascular and cerebral blood pressure changes, which may be a useful tool for clinicians to optimize medical management of HBP.

\section{Future Work}

Future work in this subject should include improvements to the segmentation algorithm in order to find statistical correlation with small blood vessels found above the circle of Willis. Steps should also be taken to ensure day 0 and day 700 MRA imaging is conducted in a manner that lines up imaging slices from both days exactly for accurate comparisons. Studying the effects of blood pressure changes in vessels above the circle of Willis prove difficult and any future work regarding these methodologies will need to consider that when imaging patients for this type of study.

The segmentation algorithm and metrics for vascular and blood pressure changes (CDF, PDF) are not limited to cerebral vasculature. These methodologies may also be used to quantify vascular changes in other end organs that are/could be sensitive to blood pressure. Understanding the blood pressure changes in those local regions may give better insight into the progression and mechanisms of various diseases. Coupling this data with information about the several types of stress patients experience in their lives may provide a better framework to manage their risk for medical complications through proper lifestyle changes and optimization of drug therapy. Data from a large pool of patients may be used to generate a working algorithm of all factors to determine those who may be at most risk. 
Several possibilities for the future work of this thesis include, but are not limited to, the following:

- Extending the presented segmentation and analysis techniques to better understand how dyslexia and autism are effected by cerebrovascular blood pressures and cerebrovascular remodeling[7, 10, 12, 35, 45, 48-79]. This process will be of a great importance to extract and quantify the changes to brain structures caused by cerebrovascular remodeling.

- Extending the functionality to be suitable for application to the heart[6, 14, 15, 80-99] to be able to diagnose heart patients (e.g., after stem cell therapy).

- Extending the functionality to study the feasibility of applying motion correction registration techniques to study kidney behavior before and after renal transplant[6, 100-124].

- Exploring the functionality and benefits of being able to detect blood pressure changes in the prostate and extending analysis techniques to be applied in the diagnosis of prostate cancer[125-136].

- Exploring the functionality and benefits of being able to detect blood pressure changes in blood vessels of the lung and extending analysis techniques to be applied in the diagnosis of lung cancer[43, 137-175]. Analysis of local changes in pulmonary structures may also reveal results beneficial for advancing the techniques used to treat diseases of the lungs.

- Exploring the functionality and benefits of being able to detect blood pressure changes in the retina and extending analysis techniques to be applied in the diagnosis of retinal diseases[176-181]. 


\section{APPENDIX I - Segmentation Initialization \& LCDG refinement}

\section{A. Sequential EM-Based Initialization}

The initial LCDG model, closely approximating a given marginal gray level distribution

F, is built using a conventional EM-algorithm, adapted to the discreet Gaussians (DG) [39, 182].

The approximation involves the following steps:

1) The distribution $\mathbf{F}$ is approximated with a mixture $\mathbf{P}_{K}$ of $K$ positive discreet Gaussians relating to a dominant mode.

2) Deviations between $\mathbf{F}$ and $\mathbf{P}_{K}$ are approximated with the alternating "subordinate" components of the LCDG as follows.

a) The positive and the negative deviations are separated and scaled up to form two seemingly "probability distributions" $\mathbf{D}^{p}$ and $\mathbf{D}^{n}$.

b) The same conventional EM algorithm is used iteratively to find a subordinate mixture of positive or negative DGs that approximates best $\mathbf{D}^{p}$ or $\mathbf{D}^{n}$, respectively (i.e., the sizes $\mathrm{C}_{p}-\mathrm{K}$ and $\mathrm{C}_{n}$ of the mixtures are found by minimizing sequentially the total absolute error between each "distribution" $\mathbf{D}^{p}$ or $\mathbf{D}^{n}$ and its mixture model by the number of the components).

c) The obtained positive and negative subordinate mixtures are scaled down and then added to the dominant mixture yielding the initial LCDG model of the size $\mathrm{C}=\mathrm{C}_{p}+\mathrm{C}_{n}$

The resulting initial LCDG has $\mathrm{K}$ dominant weights $\mathrm{w}_{p}, 1, \ldots, \mathrm{w}_{p}, K$ such that $\sum_{\mathrm{r}=1}^{K} w_{p, r}=$ 1 , and several subordinate weights of smaller values such that $\sum_{r=K+1}^{C_{p}} w_{p, r}-\sum_{l=1}^{C_{n}} w_{n, l}=0$. 


\section{B. Modified EM Algorithm for Refining LCDGs}

The initial LCDG is refined by approaching the local maximum of the log-likelihood in (3) with the EM process adapting that in [44] to the DGs. The latter extends in turn the conventional EM-process in [39] and [182] onto the alternating components.

Let $p_{w, \theta}^{[m]}(q)=\sum_{r-1}^{C_{p}} w_{p, r}^{[p]} \psi\left(\mathrm{q} \mid \theta_{\mathrm{p}, \mathrm{r}}^{[\mathrm{m}]}\right)-\sum_{l=1}^{C_{n}} w_{n, l}^{[m]} \psi\left(\mathrm{q} \mid \theta_{n, l}^{[m]}\right)$ denote the current LCDG at iteration $\mathrm{m}$. Relative contributions of each signal $\mathrm{q} \in \mathrm{Q}$ to each positive and negative $\mathrm{DG}$ at iteration $\mathrm{m}$ are specified by the respective conditional weights

$$
\pi_{p}^{[m]}(r \mid q)=\frac{w_{p, r}^{[m]} \psi\left(\mathrm{q} \mid \theta_{\mathrm{p}, \mathrm{r}}^{[\mathrm{m}]}\right)}{p_{w, \theta}^{[m]}(q)} ; \pi_{\mathrm{n}}^{[\mathrm{m}]}(l \mid q)=\frac{w_{n, l}^{[m]} \psi\left(\mathrm{q} \mid \theta_{\mathrm{n}, 1}^{[\mathrm{m}]}\right)}{p_{w, \theta}^{[m]}(q)}
$$

such that the following constraints hold:

$$
\sum_{r=1}^{C_{p}} \pi_{p}^{[m]}(r \mid q)-\sum_{l=1}^{C_{n}} \pi_{n}^{[m]}(l \mid q)=1 ; q=0, \ldots, Q-1 .
$$

The following two steps iterate until the log-likelihood is increasing and its changes become small:

E-step ${ }^{[m]}$ : Find the weights of (4) under the fixed parameters $\mathbf{w}^{[\mathrm{m}-1]}, \Theta^{[\mathrm{m}-1]}$ from the previous iteration $m-1$, and M-step ${ }^{[\mathrm{m}]}$ : Find conditional maximum likelihood estimates (MLEs) $\mathbf{w}^{[\mathrm{m}]}, \boldsymbol{\Theta}^{[\mathrm{m}]}$ by maximizing $L(\mathbf{w}, \boldsymbol{\Theta})$ under the fixed weights of (4).

Considerations closely similar to those in [39] and [182] show this process converges to a local log-likelihood maximum. The further evidence in [44] demonstrates it is a block relaxation minimization-maximization process (in a very general way, this is also shown in [182]). Let the $\log$-likelihood of (3) be rewritten in the equivalent form with the constraints of (5) as unit factors:

$$
\mathrm{L}\left(\mathbf{w}^{[m]}, \Theta^{[m]}\right)=\sum_{q=0}^{Q} f(q)\left[\sum_{r=1}^{C_{p}} \pi_{p}^{[m]}(r \mid q) \log p^{[m]}(q)-\sum_{l=1}^{C_{n}} \pi_{n}^{[m]}(l \mid q) \log p^{[m]}(q)\right]
$$


Let the terms $\log p^{[m]}(q)$ in the first and second brackets be replaced with the equal terms $\log w_{p, r}^{[m]}+\log \psi\left(q \mid \theta_{p, r}^{[m]}\right)-\log \pi_{p}^{[m]}(r \mid q)$ and $\log w_{n, l}^{[m]}+\log \psi\left(q \mid \theta_{n, l}^{[m]}\right)-\log \pi_{n}^{[m]}(l \mid q)$, respectively, which follow from (4). At the E-step, the conditional Lagrange maximization of the $\log$-likelihood of (6) under the Q restrictions of (5) results just in the weights $\pi_{p}^{[m+1]}(r \mid q)$ and $\pi_{n}^{[m+1]}(l \mid q)$ of (4) for all $r=1, \ldots, \mathrm{C}_{p} ; l=1, \ldots, \mathrm{C}_{n}$ and $q \in \mathrm{Q}$. At the M-step, the DG weights $w_{p, r}^{[m+1]}=\sum_{q \in \mathrm{Q}} f(q) \pi_{p}^{[m+1]}(r \mid q) \quad$ and $w_{n, l}^{[m+1]}=\sum_{q \in \mathrm{Q}} f(q) \pi_{n}^{[m+1]}(l \mid q)$ follow from the conditional Lagrange maximization of the loglikelihood in (6) under the restriction of (2) and the fixed conditional weights of (4). Under these latter, the conventional MLEs of the parameters of each DG stem from maximizing the loglikelihood after each difference of the cumulative Gaussians is replaced with its close approximation with the Gaussian density (below "c" stands for "p" or "n," respectively):

$$
\begin{gathered}
\mu_{c, r}^{[m+1]}=\frac{1}{w_{c, r}^{[m+1]}} \sum_{q \in \mathrm{Q}}\left(q \cdot f(q) \pi_{c}^{[m+1]}(r \mid q)\right. \\
\left(\sigma_{c, r}^{[m+1]}\right)^{2}=\frac{1}{w_{c, r}^{[m+1]}} \sum_{q \in \mathrm{Q}}\left(q-\mu_{c, i}^{[m+1]}\right)^{2} \cdot f(\mathrm{q}) \pi_{c}^{[m+1]}(r \mid q) .
\end{gathered}
$$

This modified EM-algorithm is valid until the weights $\mathrm{w}$ are strictly positive. The iterations should be terminated when the log-likelihood of (3) almost does not change or begins to decrease due to accumulation of rounding errors.

The final mixed LCDG-model $p_{C}(\mathrm{q})$ is partitioned into the K LCDG-submodels $\boldsymbol{P}_{[K]}=$ $[p(q \mid k): q \in \mathbf{Q}]$, one per class $k=1, \ldots, K$, by associating the subordinate DGs with the dominant terms so that the misclassification rate is minimal. 
APPENDIX II - Data Tables

\section{A. CDF Comparison graphs}
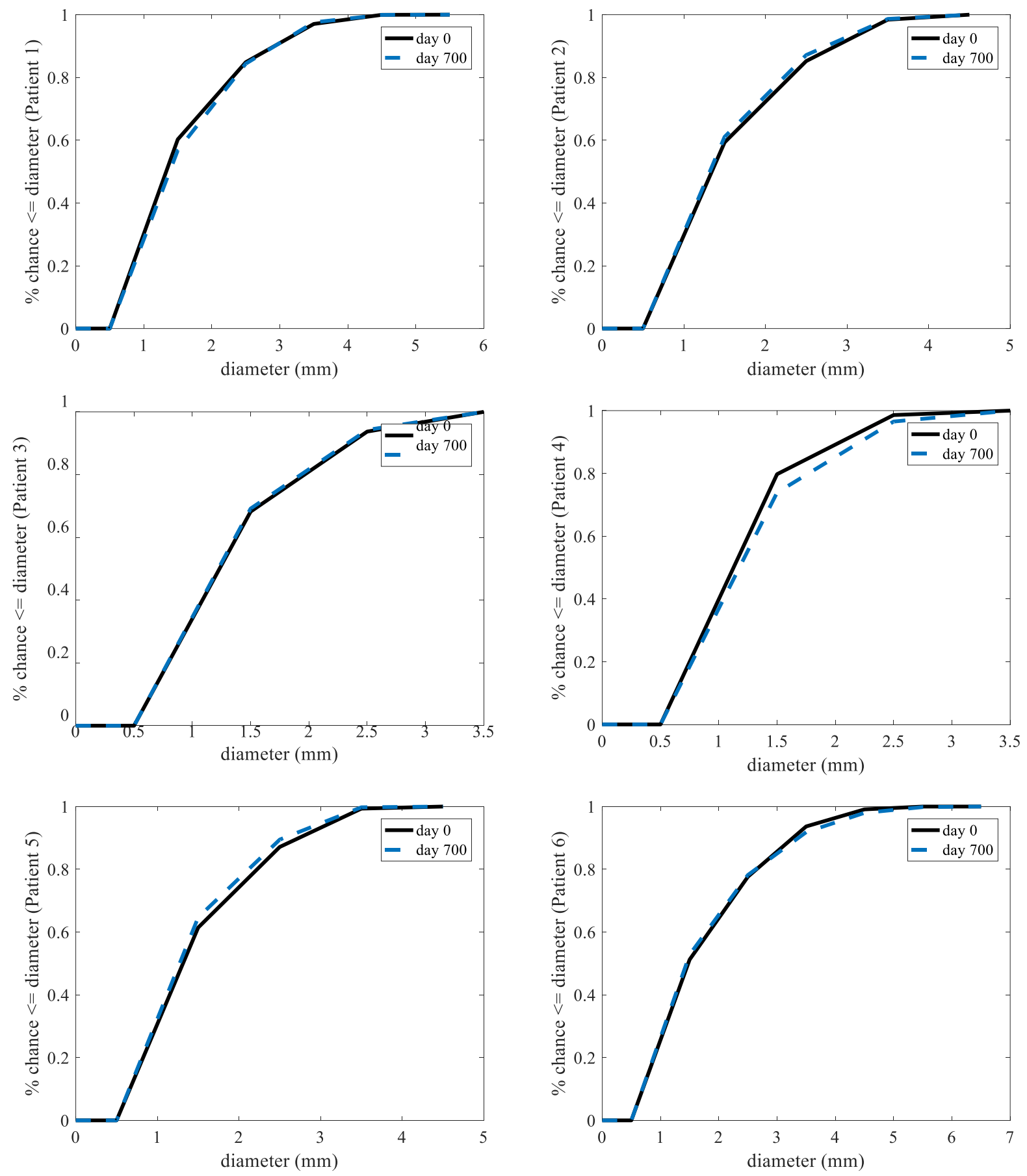

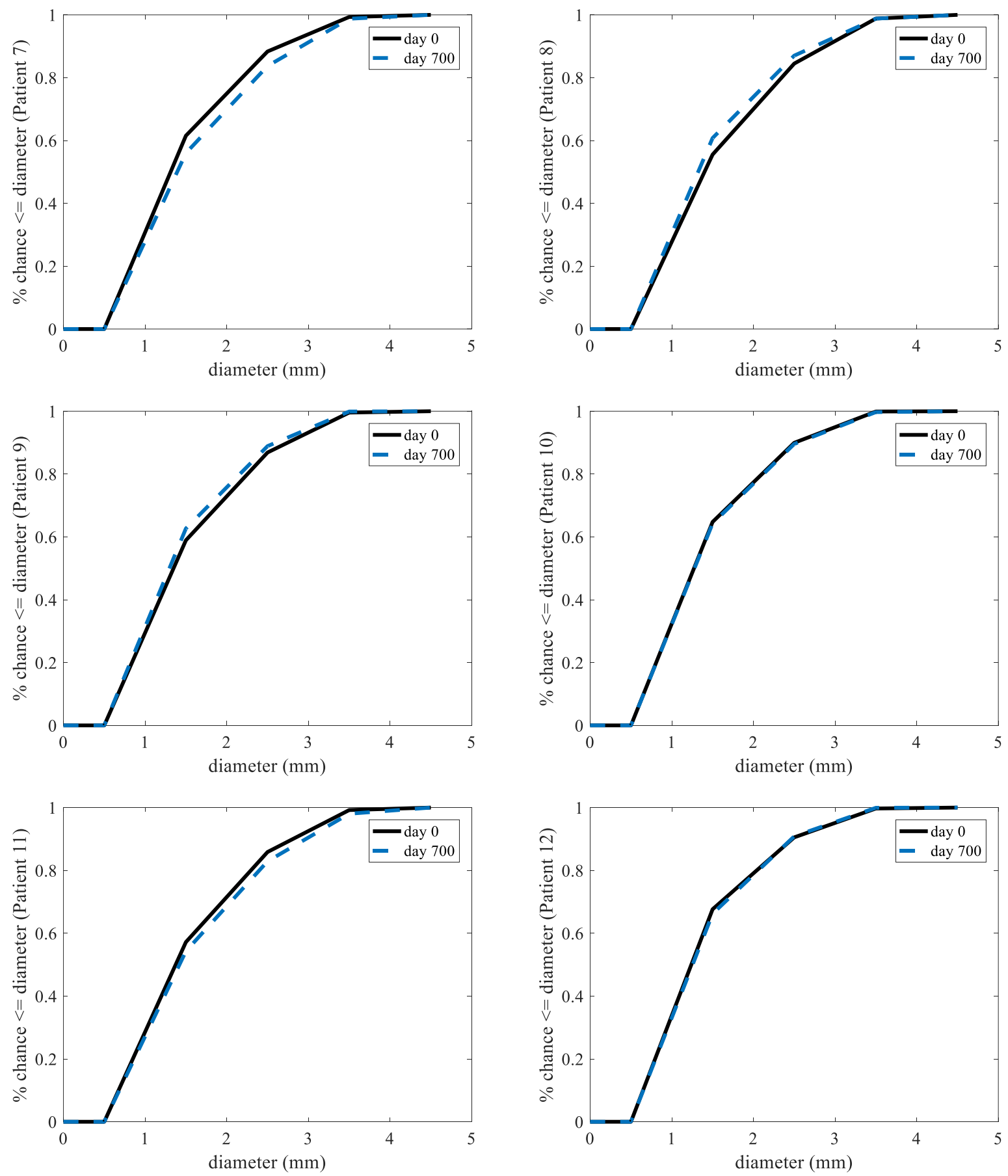

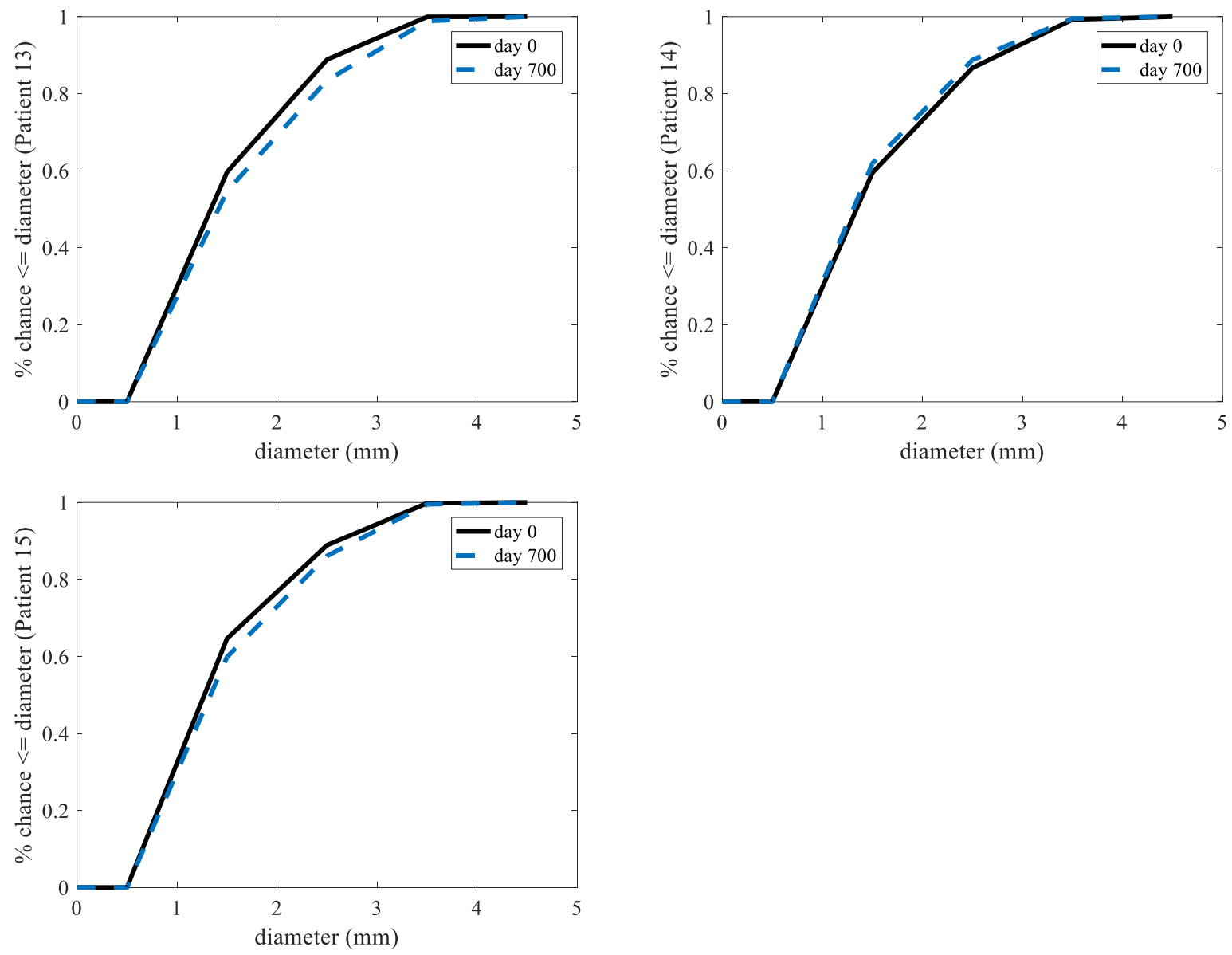


\section{B. Patient BP data}

\begin{tabular}{|r|r|r|r|r|}
\hline Patient ID & Day 0 Systolic BP & Day 0 Diastolic BP & Day 700 systolic BP & Day 700 diastolic BP \\
\hline 1 & 121.5 & 85.5 & 118.5 & 69 \\
\hline 2 & 125.5 & 88.5 & 129.5 & 93 \\
\hline 3 & 130.5 & 82.5 & 120.5 & 78 \\
\hline 4 & 118 & 80.5 & 105.5 & 69 \\
\hline 5 & 130.5 & 83 & 143.5 & 94 \\
\hline 6 & 121 & 88.5 & 130.5 & 100.5 \\
\hline 7 & 120 & 80.5 & 103.5 & 66.5 \\
\hline 8 & 129 & 78.5 & 134.5 & 86 \\
\hline 9 & 114 & 84.5 & 120 & 88 \\
\hline 10 & 119 & 84.5 & 115.5 & 76 \\
\hline 11 & 124.5 & 73 & 101 & 69 \\
\hline 12 & 133.5 & 85 & 123.5 & 79.5 \\
\hline 13 & 124 & 81.5 & 106.5 & 70.5 \\
\hline 14 & 105.5 & 85 & 132.5 & 95.5 \\
\hline 15 & 124 & 81.5 & 108.5 & 74.5 \\
\hline
\end{tabular}




\section{REFERENCES}

1. Schneiderman, N., G. Ironson, and S.D. Siegel, Stress and health: psychological, behavioral, and biological determinants. Annu. Rev. Clin. Psychol., 2005. 1: p. 607-628.

2. Keller, A., et al., Does the perception that stress affects health matter? The association with health and mortality. Health Psychology, 2012. 31(5): p. 677.

3. Control, C.f.D. About Underlying Cause of Death, 1999-2015. Centers for Disease Control and Prevention 2015; Available from: http://wonder.cdc.gov/ucd-icd10.html.

4. Kulkarni, S., et al., Stress and hypertension. WMJ: official publication of the State Medical Society of Wisconsin, 1998. 97(11): p. 34-38.

5. Mohan, S. and N.R. Campbell, Salt and high blood pressure. Clinical Science, 2009. 117(1): p. 1-11.

6. Sarnak, M.J., et al., Kidney disease as a risk factor for development of cardiovascular disease. Circulation, 2003. 108(17): p. 2154-2169.

7. Iadecola, C. and R.L. Davisson, Hypertension and cerebrovascular dysfunction. Cell metabolism, 2008. 7(6): p. 476-484.

8. van Dijk, E.J., et al., The association between blood pressure, hypertension, and cerebral white matter lesions. Hypertension, 2004. 44(5): p. 625-630.

9. Willmot, M., J. Leonardi-Bee, and P.M. Bath, High blood pressure in acute stroke and subsequent outcome. Hypertension, 2004. 43(1): p. 18-24.

10. Jennings, J.R. and A.F. Heim, From brain to behavior: hypertension's modulation of cognition and affect. International journal of hypertension, 2012. 2012.

11. Jennings, J.R., et al., Prehypertensive Blood Pressures and Regional Cerebral Blood Flow Independently Relate to Cognitive Performance in Midlife. Journal of the American Heart Association, 2017. 6(3): p. e004856.

12. Jennings, J.R. and Y. Zanstra, Is the brain the essential in hypertension? Neuroimage, 2009. 47(3): p. 914-921.

13. El-Baz, A., G. Gimel'farb, and J.S. Suri, Stochastic modeling for medical image analysis. 2015: CRC Press.

14. van der Geest, R.J. and J.H. Reiber, Quantification in cardiac MRI. Journal of Magnetic resonance imaging, 1999. 10(5): p. 602-608. 
15. Amini, A.A., J. Chen, and Y. Wang. IMAGING AND ANALYSIS FOR DETERMINATION OF CARDIOVASCULAR MECHANICS. in Biomedical Imaging: From Nano to Macro, 2007. ISBI 2007. 4th IEEE International Symposium on. 2007. IEEE.

16. Van Everdingen, K., et al., MRA flow quantification in patients with a symptomatic internal carotid artery occlusion. Stroke, 1997. 28(8): p. 1595-1600.

17. Sato, Y., et al., Three-dimensional multi-scale line filter for segmentation and visualization of curvilinear structures in medical images. Medical image analysis, 1998. 2(2): p. 143168.

18. Krissian, K., et al. Model based multiscale detection of $3 D$ vessels. in Biomedical Image Analysis, 1998. Proceedings. Workshop on. 1998. IEEE.

19. Catté, F., et al., Image selective smoothing and edge detection by nonlinear diffusion. SIAM Journal on Numerical analysis, 1992. 29(1): p. 182-193.

20. Lacoste, C., G. Finet, and I.E. Magnin. Coronary tree extraction from X-ray angiograms using marked point processes. in Biomedical Imaging: Nano to Macro, 2006. 3rd IEEE International Symposium on. 2006. IEEE.

21. Gülsün, M.A. and H. Tek. Robust vessel tree modeling. in International Conference on Medical Image Computing and Computer-Assisted Intervention. 2008. Springer.

22. Péchaud, M., R. Keriven, and G. Peyré. Extraction of tubular structures over an orientation domain. in Computer Vision and Pattern Recognition, 2009. CVPR 2009. IEEE Conference on. 2009. IEEE.

23. Li, H. and A. Yezzi, Vessels as 4-D curves: Global minimal 4-D paths to extract 3-D tubular surfaces and centerlines. IEEE transactions on medical imaging, 2007. 26(9): p. 1213-1223.

24. Benmansour, F., et al. Tubular anisotropy for $2 d$ vessel segmentation. in Computer Vision and Pattern Recognition, 2009. CVPR 2009. IEEE Conference on. 2009. IEEE.

25. Zhu, N. and A.C. Chung. Minimum average-cost path for real time $3 d$ coronary artery segmentation of ct images. in International Conference on Medical Image Computing and Computer-Assisted Intervention. 2011. Springer.

26. Lorigo, L.M., et al., Curves: Curve evolution for vessel segmentation. Medical image analysis, 2001. 5(3): p. 195-206. 
27. Xu, C. and J.L. Prince, Snakes, shapes, and gradient vector flow. IEEE Transactions on image processing, 1998. 7(3): p. 359-369.

28. Pizaine, G., et al. Implicit medial representation for vessel segmentation. in SPIE Medical Imaging. 2011. International Society for Optics and Photonics.

29. McInerney, T. and D. Terzopoulos. Medical image segmentation using topologically adaptable snakes. in Computer Vision, Virtual Reality and Robotics in Medicine. 1995. Springer.

30. Caselles, V., R. Kimmel, and G. Sapiro, Geodesic active contours. International journal of computer vision, 1997. 22(1): p. 61-79.

31. Wink, O., W.J. Niessen, and M.A. Viergever, Fast delineation and visualization of vessels in 3-D angiographic images. IEEE transactions on medical imaging, 2000. 19(4): p. 337346.

32. Deschamps, T. and L.D. Cohen. Fast extraction of tubular and tree 3D surfaces with front propagation methods. in Pattern Recognition, 2002. Proceedings. 16th International Conference on. 2002. IEEE.

33. Manniesing, R., et al., Level set based cerebral vasculature segmentation and diameter quantification in CT angiography. Medical image analysis, 2006. 10(2): p. 200-214.

34. Holtzman-Gazit, M., et al., Segmentation of thin structures in volumetric medical images. IEEE Transactions on image processing, 2006. 15(2): p. 354-363.

35. Forkert, N.D., et al. Direction-dependent level set segmentation of cerebrovascular structures. in SPIE Medical Imaging. 2011. International Society for Optics and Photonics.

36. El-Baz, A., et al., Precise segmentation of 3-D magnetic resonance angiography. IEEE Transactions on Biomedical Engineering, 2012. 59(7): p. 2019-2029.

37. Chung, A.C. and J.A. Noble. Statistical 3D vessel segmentation using a Rician distribution. in International Conference on Medical Image Computing and Computer-Assisted Intervention. 1999. Springer.

38. Wilson, D.L. and J.A. Noble, An adaptive segmentation algorithm for time-of-flight MRA data. IEEE transactions on medical imaging, 1999. 18(10): p. 938-945.

39. Webb, A.R., Statistical pattern recognition. 2003: John Wiley \& Sons.

40. Nain, D., A. Yezzi, and G. Turk, Vessel segmentation using a shape driven flow. Medical Image Computing and Computer-Assisted Intervention-MICCAI 2004, 2004: p. 51-59. 
41. Law, M.W. and A.C. Chung. A deformable surface model for vascular segmentation. in International Conference on Medical Image Computing and Computer-Assisted Intervention. 2009. Springer.

42. Gan, R., et al. Vascular segmentation in three-dimensional rotational angiography based on maximum intensity projections. in Biomedical Imaging: Nano to Macro, 2004. IEEE International Symposium on. 2004. IEEE.

43. $\mathrm{Hu}, \mathrm{S}$, E.A. Hoffman, and J.M. Reinhardt, Automatic lung segmentation for accurate quantitation of volumetric X-ray CT images. IEEE transactions on medical imaging, 2001. 20(6): p. 490-498.

44. Gimel'farb, G., A.A. Farag, and A. El-Baz. Expectation-Maximization for a linear combination of Gaussians. in Pattern Recognition, 2004. ICPR 2004. Proceedings of the 17th International Conference on. 2004. IEEE.

45. Sabry, M., et al., A fast automatic method for 3D volume segmentation of the human cerebrovascular, in CARS 2002 Computer Assisted Radiology and Surgery. 2002, Springer. p. 382-387.

46. Suzuki, K., Computerized segmentation of organs by means of geodesic active-contour level-set algorithm, in Multi Modality State-of-the-Art Medical Image Segmentation and Registration Methodologies. 2011, Springer. p. 103-128.

47. Itai, Y., et al., Development of a voxel-matching technique for substantial reduction of subtraction artifacts in temporal subtraction images obtained from thoracic MDCT. Journal of digital imaging, 2010. 23(1): p. 31-38.

48. Alansary, A., et al., MAP-based framework for segmentation of MR brain images based on visual appearance and prior shape. MIDAS J, 2013. 1: p. 1.

49. El-Baz, A., et al. A new CAD system for early diagnosis of dyslexic brains. in Image Processing, 2008. ICIP 2008. 15th IEEE International Conference on. 2008. IEEE.

50. Elnakib, A., et al., Analysis of 3D Corpus Callosum Images in the Brains of Autistic Individuals, in Handbook of Research on Trends in the Diagnosis and Treatment of Chronic Conditions. 2016, IGI Global. p. 159-184.

51. Elnakib, A., et al. Image-based detection of corpus callosum variability for more accurate discrimination between autistic and normal brains. in Image Processing (ICIP), 2010 17th IEEE International Conference on. 2010. IEEE. 
52. Fahmi, R., et al., Structural MRI-based discrimination between autistic and typically developing brain. Proc. of Computer Assisted Radiology and Surgery (CARS’07), Berlin, Germany, 2007: p. 24-26.

53. Mostapha, M., et al. Atlas-based approach for the segmentation of infant dti mr brain images. in Biomedical Imaging (ISBI), 2014 IEEE 11th International Symposium on. 2014. IEEE.

54. Nitzken, M., M. Casanova, and A. El-Baz. Spharm analysis of the brain cortex for diagnosing dyslexia. in Proc IEEE International Symposium on Biomendical Imaging: From Nano to Macro (ISBI'14), Bejing, China. 2014.

55. Nitzken, M., et al. 3D shape analysis of the brain cortex with application to autism. in Biomedical Imaging: From Nano to Macro, 2011 IEEE International Symposium on. 2011. IEEE.

56. Nitzken, M.J., et al., Shape analysis of the human brain: a brief survey. IEEE journal of biomedical and health informatics, 2014. 18(4): p. 1337-1354.

57. El-Baz, A., et al., Accurate automated detection of autism related corpus callosum abnormalities. Journal of medical systems, 2011. 35(5): p. 929-939.

58. Elnakib, A., et al., Autism diagnostics by 3D shape analysis of the corpus callosum. Machine Learning in Computer-aided Diagnosis: Medical Imaging Intelligence and Analysis, 2012. 15: p. 315-335.

59. Elnakib, A., et al. Autism diagnostics by centerline-based shape analysis of the corpus callosum. in Biomedical Imaging: From Nano to Macro, 2011 IEEE International Symposium on. 2011. IEEE.

60. Elnakib, A., et al., Dyslexia diagnostics by 3-D shape analysis of the corpus callosum. IEEE Transactions on Information Technology in Biomedicine, 2012. 16(4): p. 700-708.

61. Elnakib, A., et al. Dyslexia diagnostics by centerline-based shape analysis of the corpus callosum. in Pattern Recognition (ICPR), 2010 20th International Conference on. 2010. IEEE.

62. Mostapha, M., et al. A statistical framework for the classification of infant dt images. in Image Processing (ICIP), 2014 IEEE International Conference on. 2014. IEEE. 
63. El-Baz, A., et al., Automatic cerebrovascular segmentation by accurate probabilistic modeling of TOF-MRA images. Medical Image Computing and Computer-Assisted Intervention-MICCAI 2005, 2005: p. 34-42.

64. Dombroski, B., et al., Gyral window mapping of typical cortical folding using MRI. Translational neuroscience, 2011. 2(2): p. 142-147.

65. Elnakib, A., et al., Magnetic resonance imaging findings for dyslexia: a review. Journal of biomedical nanotechnology, 2014. 10(10): p. 2778-2805.

66. Ismail, M., et al. Detection of white matter abnormalities in mr brain images for diagnosis of autism in children. in Biomedical Imaging (ISBI), 2016 IEEE 13th International Symposium on. 2016. IEEE.

67. Ismail, M., et al. Segmentation of infant brain MR images based on adaptive shape prior and higher-order MGRF. in Image Processing (ICIP), 2015 IEEE International Conference on. 2015. IEEE.

68. Alansary, A., et al., Infant Brain Extraction in T1-weighted MR Images using BET and Refinement using LCDG and MGRF Models. IEEE journal of biomedical and health informatics, 2016. 20(3): p. 925-935.

69. Alansary, A., et al. An integrated geometrical and stochastic approach for accurate infant brain extraction. in Image Processing (ICIP), 2014 IEEE International Conference on. 2014. IEEE.

70. El-Baz, A., et al. A new image analysis approach for automatic classification of autistic brains. in Biomedical Imaging: From Nano to Macro, 2007. ISBI 2007. 4th IEEE International Symposium on. 2007. IEEE.

71. Nitzken, M., et al., Shape-based detection of cortex variability for more accurate discrimination between autistic and normal brains, in Multi Modality State-of-the-Art Medical Image Segmentation and Registration Methodologies. 2011, Springer. p. 161-185.

72. Casanova, M.F., et al., Focal cortical dysplasias in autism spectrum disorders. Acta neuropathologica communications, 2013. 1(1): p. 67.

73. El-Baz, A., et al., Autism diagnostics by $3 D$ texture analysis of cerebral white matter gyrifications. Medical Image Computing and Computer-Assisted Intervention-MICCAI 2007, 2007: p. 882-890. 
74. Ismail, M.M., et al., Studying autism spectrum disorder with structural and diffusion magnetic resonance imaging: a survey. Frontiers in human neuroscience, 2016. 10.

75. El-Baz, A., et al., An MRI-based diagnostic framework for early diagnosis of dyslexia. International Journal of Computer Assisted Radiology and Surgery, 2008. 3(3-4): p. 181.

76. El-Baz, A., et al. Dyslexia diagnostics by 3D texture analysis of cerebral white matter gyrifications. in Pattern Recognition, 2008. ICPR 2008. 19th International Conference on. 2008. IEEE.

77. El-Baz, A., et al., A new adaptive probabilistic model of blood vessels for segmenting MRA images. Medical Image Computing and Computer-Assisted Intervention-MICCAI 2006, 2006: p. 799-806.

78. Dombroski, B., et al., Cortical surface complexity in a population-based normative sample. Translational Neuroscience, 2014. 5(1): p. 17-24.

79. El-Baz, A., et al. Probabilistic modeling of blood vessels for segmenting MRA images. in Pattern Recognition, 2006. ICPR 2006. 18th International Conference on. 2006. IEEE.

80. Beache, G.M., et al., Fully automated framework for the analysis of myocardial first-pass perfusion MR images. Medical physics, 2014. 41(10).

81. Elnakib, A., et al. A new framework for automated segmentation of left ventricle wall from contrast enhanced cardiac magnetic resonance images. in Image Processing (ICIP), 2011 18th IEEE International Conference on. 2011. IEEE.

82. Elnakib, A., et al., New automated Markov-Gibbs random field based framework for myocardial wall viability quantification on agent enhanced cardiac magnetic resonance images. The international journal of cardiovascular imaging, 2012. 28(7): p. 1683-1698.

83. Elnakib, A., et al., Intramyocardial strain estimation from cardiac cine MRI. International journal of computer assisted radiology and surgery, 2015. 10(8): p. 1299-1312.

84. Elnakib, A., et al. Validating a new methodology for strain estimation from cardiac cine MRI. in AIP Conference Proceedings. 2013. AIP.

85. Khalifa, F., et al. A new nonrigid registration framework for improved visualization of transmural perfusion gradients on cardiac first-pass perfusion MRI. in Biomedical Imaging (ISBI), 2012 9th IEEE International Symposium on. 2012. IEEE. 
86. Nitzken, M.J., A.S. El-Baz, and G.M. Beache, Markov-Gibbs Random Field Model for Improved Full-Cardiac Cycle Strain Estimation from Tagged CMR. Journal of Cardiovascular Magnetic Resonance, 2012. 14(1): p. P258.

87. Sliman, H., et al. Accurate segmentation framework for the left ventricle wall from cardiac cine MRI. in AIP Conference Proceedings. 2013. AIP.

88. Elnakib, A., et al. A novel laplace-based method to estimate the strain from cine cardiac magnetic resonance images. in Proc. IEEE Int. Conf. Image Process.,(ICIP'13). 2013.

89. Elnakib, A., et al. A new framework for automated identification of pathological tissues in contrast enhanced cardiac magnetic resonance images. in Biomedical Imaging: From Nano to Macro, 2011 IEEE International Symposium on. 2011. IEEE.

90. Khalifa, F., et al. A new shape-based framework for the left ventricle wall segmentation from cardiac first-pass perfusion MRI. in Biomedical Imaging (ISBI), 2013 IEEE 10th International Symposium on. 2013. IEEE.

91. Khalifa, F., et al. A new nonrigid registration approach for motion correction of cardiac first-pass perfusion MRI. in Image Processing (ICIP), 2012 19th IEEE International Conference on. 2012. IEEE.

92. Khalifa, F., et al. A novel CAD system for analyzing cardiac first-pass MR images. in Pattern Recognition (ICPR), 2012 21st International Conference on. 2012. IEEE.

93. Khalifa, F., et al., Accurate automatic analysis of cardiac cine images. IEEE Transactions on Biomedical Engineering, 2012. 59(2): p. 445-455.

94. Nitzken, M., et al. Accurate modeling of tagged CMR 3D image appearance characteristics to improve cardiac cycle strain estimation. in Image Processing (ICIP), 2012 19th IEEE International Conference on. 2012. IEEE.

95. Sliman, H., et al., Assessment of Myocardial Function from Cine Cardiac MRI Using a Novel 4D Tracking Approach. Journal of Computer Science and System Biology, 2014. 7: p. 169-173.

96. Sliman, H., et al. A novel 4D PDE-based approach for accurate assessment of myocardium function using cine cardiac magnetic resonance images. in Image Processing (ICIP), 2014 IEEE International Conference on. 2014. IEEE. 
97. Sliman, H., et al. A new segmentation-based tracking framework for extracting the left ventricle cavity from cine cardiac MRI. in Proceedings of the IEEE international conference on image processing (ICIP'13), Melbourne, Australia. 2013.

98. Sliman, H., et al., Myocardial borders segmentation from cine MR images using bidirectional coupled parametric deformable models. Medical physics, 2013. 40(9).

99. Mulvany, M.J., Small artery remodeling and significance in the development of hypertension. Physiology, 2002. 17(3): p. 105-109.

100. Khalifa, F., et al. A random forest-based framework for 3D kidney segmentation from dynamic contrast-enhanced CT images. in Image Processing (ICIP), 2016 IEEE International Conference on. 2016. IEEE.

101. Khalifa, F., et al. Kidney segmentation from CT images using a 3D NMF-guided active contour model. in Biomedical Imaging (ISBI), 2016 IEEE 13th International Symposium on. 2016. IEEE.

102. Rudra, A.K., et al., Kidney segmentation using graph cuts and pixel connectivity. Pattern Recognition Letters, 2013. 34(13): p. 1470-1475.

103. Shehata, M., et al. A novel framework for automatic segmentation of kidney from DW-MRI. in Biomedical Imaging (ISBI), 2015 IEEE 12th International Symposium on. 2015. IEEE.

104. Shehata, M., et al. A level set-based framework for 3D kidney segmentation from diffusion MR images. in Image Processing (ICIP), 2015 IEEE International Conference on. 2015. IEEE.

105. Shehata, M., et al., An appearance-guided deformable model for 4D kidney segmentation using diffusion MRI. Biomedical Image Segmentation: Advances and Trends, 2016: p. 271.

106. Shehata, M., et al. A Promising Non-invasive CAD System for Kidney Function Assessment. in International Conference on Medical Image Computing and Computer-Assisted Intervention. 2016. Springer.

107. El-Baz, A., et al. Image analysis of renal DCE MRI for the detection of acute renal rejection. in Pattern Recognition, 2006. ICPR 2006. 18th International Conference on. 2006. IEEE.

108. Khalifa, F., et al., Dynamic contrast-enhanced MRI-based early detection of acute renal transplant rejection. IEEE transactions on medical imaging, 2013. 32(10): p. 1910-1927. 
109. Khalifa, F., et al., Non-invasive image-based approach for early detection of acute renal rejection. Medical Image Computing and Computer-Assisted Intervention-MICCAI 2010, 2010: p. 10-18.

110. Shehata, M., et al. 3D diffusion MRI-based CAD system for early diagnosis of acute renal rejection. in Biomedical Imaging (ISBI), 2016 IEEE 13th International Symposium on. 2016. IEEE.

111. Khalifa, F., et al., A New Image-Based Framework for Analyzing Cine Images, in Multi Modality State-of-the-Art Medical Image Segmentation and Registration Methodologies. 2011, Springer. p. 69-98.

112. El-Baz, A., et al., Application of deformable models for the detection of acute renal rejection. Deformable models, 2007: p. 293-333.

113. El-Baz, A., G. Gimel'farb, and M.A. El-Ghar. New motion correction models for automatic identification of renal transplant rejection. in International Conference on Medical Image Computing and Computer-Assisted Intervention. 2007. Springer.

114. El-Ghar, M.A., et al., Computer aided detection of acute renal allograft dysfunction using dynamic contrast enhanced MRI. The Egyptian Journal of Radiology and Nuclear Medicine, 2011. 42(3): p. 443-449.

115. Farag, A., et al. A framework for the detection of acute renal rejection with dynamic contrast enhanced magnetic resonance imaging. in Biomedical Imaging: Nano to Macro, 2006. 3rd IEEE International Symposium on. 2006. IEEE.

116. Khalifa, F., et al., A comprehensive non-invasive framework for automated evaluation of acute renal transplant rejection using DCE-MRI. NMR in Biomedicine, 2013. 26(11): p. 1460-1470.

117. Mostapha, M., et al., Computer-aided diagnosis systems for acute renal transplant rejection: Challenges and methodologies, in Abdomen and Thoracic Imaging. 2014, Springer. p. 1-35.

118. Shehata, M., et al. A new non-invasive approach for early classification of renal rejection types using diffusion-weighted MRI. in Image Processing (ICIP), 2016 IEEE International Conference on. 2016. IEEE.

119. Shehata, M.N.M.I., A non-invasive diagnostic system for early assessment of acute renal transplant rejection. 2016. 
120. Yuksel, S.E., et al. Automatic detection of renal rejection after kidney transplantation. in International Congress Series. 2005. Elsevier.

121. El-Baz, A., et al., A new CAD system for the evaluation of kidney diseases using DCEMRI. Medical Image Computing and Computer-Assisted Intervention-MICCAI 2006, 2006: p. 446-453.

122. Mostapha, M., et al. Dynamic MRI-based computer aided diagnostic systems for early detection of kidney transplant rejection: A survey. in AIP Conference Proceedings. 2013. AIP.

123. M. Shehata, F.K., A. Soliman, M. Abou El-Ghar, A. C. Dwyer, and A. El-Baz, Assessment of Renal Transplant Using Image and Clinical-based Biomarkers. Proceedings of 13th Annual Scientific Meeting of American Society for Diagnostics and Interventional Nephrology (ASDIN'17).

124. F. Khalifa, M.S., A. Soliman, M. Abou El-Ghar, T. El-Diasty, A. C. Dwyer, M. El-Melegy, R. Keynton, A. El-Baz, A GENERALIZED MRI-BASED CAD SYSTEM FOR FUNCTIONAL ASSESSMENT OF RENAL TRANSPLANT Proceedings of International Symposium on Biomedical Imaging: From Nano to Macro (ISBI'17).

125. Firjani, A., et al., A diffusion-weighted imaging based diagnostic system for early detection of prostate cancer. Journal of Biomedical Science and Engineering, 2013. 6(03): p. 346.

126. Firjani, A., et al. A new 3D automatic segmentation framework for accurate segmentation of prostate from DCE-MRI. in Biomedical Imaging: From Nano to Macro, 2011 IEEE International Symposium on. 2011. IEEE.

127. Firjani, A., et al. 3D automatic approach for precise segmentation of the prostate from diffusion-weighted magnetic resonance imaging. in Image Processing (ICIP), 2011 18th IEEE International Conference on. 2011. IEEE.

128. Firjani, A., et al. A novel image-based approach for early detection of prostate cancer. in Image Processing (ICIP), 2012 19th IEEE International Conference on. 2012. IEEE.

129. Firjany, A., et al. Novel stochastic framework for accurate segmentation of prostate in dynamic contrast enhanced MRI. in International Workshop on Prostate Cancer Imaging. 2010. Springer.

130. McClure, P., et al., In-vitro and in-vivo diagnostic techniques for prostate cancer: A review. Journal of biomedical nanotechnology, 2014. 10(10): p. 2747-2777. 
131. McClure, P., et al., A novel NMF guided level-set for DWI prostate segmentation. Journal of Computer Science \& Systems Biology, 2014. 7(6): p. 1.

132. Reda, I., et al. A new NMF-Autoencoder based CAD system for early diagnosis of prostate cancer. in Biomedical Imaging (ISBI), 2016 IEEE 13th International Symposium on. 2016. IEEE.

133. Firjani, A., A. Elmaghraby, and A. El-Baz. MRI-based diagnostic system for early detection of prostate cancer. in Biomedical Sciences and Engineering Conference (BSEC), 2013. 2013. IEEE.

134. Firjani, A., et al. A novel 3D segmentation approach for segmenting the prostate from dynamic contrast enhanced MRI using current appearance and learned shape prior. in Signal Processing and Information Technology (ISSPIT), 2010 IEEE International Symposium on. 2010. IEEE.

135. Firjani, A., et al. A new 3D automatic segmentation framework for accurate extraction of prostate from diffusion imaging. in Biomedical Sciences and Engineering Conference (BSEC), 2011. 2011. IEEE.

136. Firjani, A., et al. Non-invasive image-based approach for early detection of prostate cancer. in Developments in E-systems Engineering (DeSE), 2011. 2011. IEEE.

137. Abdollahi, B., et al. A novel 3D joint MGRF framework for precise lung segmentation. in International Workshop on Machine Learning in Medical Imaging. 2012. Springer.

138. Abdollahi, B., et al. A novel gaussian scale space-based joint MGRF framework for precise lung segmentation. in Image Processing (ICIP), 2012 19th IEEE International Conference on. 2012. IEEE.

139. Ali, A.M., A.S. El-Baz, and A.A. Farag. A novel framework for accurate lung segmentation using graph cuts. in Biomedical Imaging: From Nano to Macro, 2007. ISBI 2007. 4th IEEE International Symposium on. 2007. IEEE.

140. El-Ba, A., et al. A new stochastic framework for accurate lung segmentation. in International Conference on Medical Image Computing and Computer-Assisted Intervention. 2008. Springer.

141. El-Baz, A., et al., Lung imaging data analysis. International journal of biomedical imaging, 2013. 2013. 
142. El-Baz, A., et al., Computer-aided diagnosis systems for lung cancer: challenges and methodologies. International journal of biomedical imaging, 2013. 2013.

143. El-Baz, A., et al., Automatic detection of $2 D$ and $3 D$ lung nodules in chest spiral CT scans. International journal of biomedical imaging, 2013. 2013.

144. El-Baz, A., et al. A framework for automatic segmentation of lung nodules from low dose chest CT scans. in Pattern Recognition, 2006. ICPR 2006. 18th International Conference on. 2006. IEEE.

145. El-Baz, A., et al. A unified approach for detection, visualization, and identification of lung abnormalities in chest spiral CT scans. in International Congress Series. 2003. Elsevier.

146. El-Baz, A., et al. Detection, visualization and identification of lung abnormalities in chest spiral CT scan: Phase-I. in International conference on biomedical engineering, Cairo, Egypt. 2002.

147. El-Baz, A., et al., Toward early diagnosis of lung cancer. Medical Image Computing and Computer-Assisted Intervention-MICCAI 2009, 2009: p. 682-689.

148. El-Baz, A., et al., Automatic analysis of 3D low dose CT images for early diagnosis of lung cancer. Pattern Recognition, 2009. 42(6): p. 1041-1051.

149. El-Baz, A., et al., 3D MGRF-based appearance modeling for robust segmentation of pulmonary nodules in 3D LDCT chest images. Lung imaging and computer aided diagnosis, 2011. 3: p. 51-63.

150. El-Baz, A., et al., Appearance analysis for the early assessment of detected lung nodules. Lung imaging and computer aided diagnosis, 2011. 17: p. 395-404.

151. El-Baz, A., et al. A novel approach for accurate estimation of the growth rate of the detected lung nodules. in Proceedings of the 1st International Workshop on Pulmonary Image Analysis. 2008.

152. El-Baz, A., et al. Appearance-based diagnostic system for early assessment of malignant lung nodules. in Image Processing (ICIP), 2012 19th IEEE International Conference on. 2012. IEEE.

153. El-Baz, A., et al. Appearance analysis for diagnosing malignant lung nodules. in Biomedical Imaging: From Nano to Macro, 2010 IEEE International Symposium on. 2010. IEEE. 
154. El-Baz, A., et al. A new approach for automatic analysis of $3 D$ low dose CT images for accurate monitoring the detected lung nodules. in Pattern Recognition, 2008. ICPR 2008. 19th International Conference on. 2008. IEEE.

155. El-Baz, A., et al. A novel approach for automatic follow-up of detected lung nodules. in Image Processing, 2007. ICIP 2007. IEEE International Conference on. 2007. IEEE.

156. El-Baz, A., et al. A new CAD system for early diagnosis of detected lung nodules. in Image Processing, 2007. ICIP 2007. IEEE International Conference on. 2007. IEEE.

157. El-Baz, A., et al. Promising results for early diagnosis of lung cancer. in Biomedical Imaging: From Nano to Macro, 2008. ISBI 2008. 5th IEEE International Symposium on. 2008. IEEE.

158. El-Baz, A., et al. A Framework for Unsupervised Segmentation of Lung Tissues from Low Dose Computed Tomography Images. in BMVC. 2008.

159. El-Baz, A., et al., Three-dimensional shape analysis using spherical harmonics for early assessment of detected lung nodules. Lung Imaging and Computer Aided Diagnosis, 2011. 19: p. 421-438.

160. El-Baz, A., et al. A novel shape-based diagnostic approach for early diagnosis of lung nodules. in Biomedical Imaging: From Nano to Macro, 2011 IEEE International Symposium on. 2011. IEEE.

161. El-Baz, A., et al., Validation of a new imaged-based approach for the accurate estimating of the growth rate of detected lung nodules using real computed tomography images and elastic phantoms generated by state-of-theart microfluidics technology. Lung Imaging and Computer Aided Diagnosis, 2011. 18: p. 405-420.

162. El-Baz, A., et al., Elastic phantoms generated by microfluidics technology: Validation of an imaged-based approach for accurate measurement of the growth rate of lung nodules. Biotechnology journal, 2011. 6(2): p. 195-203.

163. El-Baz, A., et al. Early assessment of malignant lung nodules based on the spatial analysis of detected lung nodules. in Biomedical Imaging (ISBI), 2012 9th IEEE International Symposium on. 2012. IEEE.

164. El-Baz, A., et al. Non-rigid registration techniques for automatic follow-up of lung nodules. in International Congress Series. 2005. Elsevier. 
165. El-Bazl, A., et al. Automatic identification of lung abnormalities in chest spiral CT scans. in Acoustics, Speech, and Signal Processing, 2003. Proceedings.(ICASSP'03). 2003 IEEE International Conference on. 2003. IEEE.

166. Farag, A., et al., Automatic detection and recognition of lung abnormalities in helical CT images using deformable templates. Medical Image Computing and Computer-Assisted Intervention-MICCAI 2004, 2004: p. 856-864.

167. Farag, A.A., et al. Detection and recognition of lung abnormalities using deformable templates. in Pattern Recognition, 2004. ICPR 2004. Proceedings of the 17th International Conference on. 2004. IEEE.

168. Soliman, A., et al. Segmentationof pathological lungs from CT chest images. in Image Processing (ICIP), 2015 IEEE International Conference on. 2015. IEEE.

169. Soliman, A., et al. Segmentation of lung region based on using parallel implementation of joint MGRF: Validation on $3 D$ realistic lung phantoms. in Biomedical Imaging (ISBI), 2013 IEEE 10th International Symposium on. 2013. IEEE.

170. Soliman, A., et al. Performance evaluation of an automatic MGRF-based lung segmentation approach. in AIP Conference Proceedings. 2013. AIP.

171. Soliman, A., et al. An ISO-surfaces based local deformation handling framework of lung tissues. in Biomedical Imaging (ISBI), 2016 IEEE 13th International Symposium on. 2016. IEEE.

172. Soliman, A., et al., Accurate Lungs Segmentation on CT Chest Images by Adaptive Appearance-Guided Shape Modeling. IEEE Transactions on Medical Imaging, 2017. 36(1): p. 263-276.

173. Soliman, A., et al. Detection of lung injury using 4D-CT chest images. in Biomedical Imaging (ISBI), 2016 IEEE 13th International Symposium on. 2016. IEEE.

174. Soliman, A., et al. Image-based CAD system for accurate identification of lung injury. in Image Processing (ICIP), 2016 IEEE International Conference on. 2016. IEEE.

175. Suri, J.S., Lung imaging and computer aided diagnosis. 2011: CRC Press.

176. Ahmed ElTanboly, A.P., Ahmed Shalaby, Andy E. Switala, Omar Helmy, Shlomit Schaal, and Ayman El-Baz, An automated approach for early detection of diabetics retinopathy. Frontier in Bioscience. 
177. El Tanboly, A., et al. A novel automatic segmentation of healthy and diseased retinal layers from OCT scans. in Image Processing (ICIP), 2016 IEEE International Conference on. 2016. IEEE.

178. ElTanboly, A., et al., A computer aided diagnostic system for detecting diabetic retinopathy in optical coherence tomography images. Medical Physics, 2016.

179. Neyer, T., et al., A Novel Automated Method for the Objective Quantification of Retinal Layers Based on Spectral Domain Optical Coherence Tomography (SD-OCT) Imaging Reveals Sequential Changes in the Normal Retina with Age. Investigative Ophthalmology \& Visual Science, 2016. 57(12): p. 5943-5943.

180. Schaal, S., et al., A novel automated method for the objective quantification of Retinal layers reveals sequential changes that occur in the Normal retina with age.

181. Schaal, S., et al., Subtle Early Changes in Diabetic Retinas Revealed by a Novel Method that Automatically Quantifies Spectral Domain Optical Coherence Tomography (SD-OCT) Images. Investigative Ophthalmology \& Visual Science, 2016. 57(12): p. 6324-6324.

182. Friedman, J., T. Hastie, and R. Tibshirani, The elements of statistical learning. Vol. 1. 2001: Springer series in statistics Springer, Berlin. 